\title{
Longitudinal changes in maternal circulating microRNAs as potential biomarkers of specific events during healthy pregnancy
}

Erika Chavira-Suárez ( $\nabla$ erika@bq.unam.mx)

Universidad Nacional Autónoma de México https://orcid.org/0000-0002-1012-2760

Alma Lilia Hernández-Olvera

Universidad Nacional Autónoma de México: Universidad Nacional Autonoma de Mexico

Mariana Flores-Torres

Instituto Nacional de Medicina Genomica

Karen Rubí Celaya-Cruz

Instituto Nacional de Medicina Genomica

\section{Sofía Gitler}

Instituto Nacional de Medicina Genomica

Juan Carlos De la Cerda-Ángeles

Secretaría de Salud de Mexico: Secretaria de Salud de Mexico

Nidia Carolina Espinosa-Maldonado

Instituto Nacional de Medicina Genomica

Carlos Fabián Flores-Jasso

Instituto Nacional de Medicina Genomica

Humberto Gutiérrez

Instituto Nacional de Medicina Genomica

Felipe Vadillo-Ortega

Universidad Nacional Autonoma de Mexico https://orcid.org/0000-0001-7137-4200

\section{Research Article}

Keywords: circulating microRNAs, pregnancy, computational genomics, fetal growth

Posted Date: September 28th, 2021

DOI: https://doi.org/10.21203/rs.3.rs-928551/v1

License: (c) (i) This work is licensed under a Creative Commons Attribution 4.0 International License.

Read Full License 


\section{Abstract}

\section{Background}

Novel high-resolution tools for pregnancy monitoring, including early detection of prenatal disorders, are needed. Changes in circulating microRNAs (c-miRNAs) during pregnancy could potentially inform about the functional status of the mother, the placenta and/or the fetus. However, whether c-miRNA profiles actually reflect distinct pregnancy-specific events at all stages remains unclear.

\section{Methods}

Longitudinal large-scale RNAseq c-miRNA profiles at early, middle and late pregnancy, and after birth derived from eight women with healthy term pregnancies ( $n=32$ plasma samples) were compared against corresponding circulating profiles derived from age-matched non-pregnant women $(n=10)$. Data

of fetal sex and growth indicators obtained during pregnancy evolution of the same women, were used to identify specific c-miRNA correlates in circulation.

Results

1449 c-miRNAs were detected in circulation in both pregnant and non-pregnant women with only 48 cmiRNAs differentially expressed relative to non-pregnant controls in at least one of the four studied stages (FDR < 0.05). Surprisingly, c-miRNA subpopulations with reported prominent expression in various pregnancy-associated compartments (placenta, amniotic fluid, umbilical cord plasma and breast milk) were found collectively under-expressed in maternal circulation throughout pregnancy $(p<0.05)$. Furthermore, we found a bias in global miRNAs expression in direct association with fetal sex right from the first trimester, in addition to a specific c-miRNA signature of fetal growth $(R=0.7, p<0.01)$.

\section{Conclusion}

Our results demonstrate the existence of temporal changes in c-miRNAs populations associated to distinct aspects of pregnancy, including correlates of placental function and lactation, as well as fetal gender and growth, revealing a wider potential of c-miRNAs as biomarkers of specific aspects of maternal health and fetal growth.

\section{Background}

MicroRNAs (miRNAs) are a class of non-coding short sequence ribonucleic acids (ncRNAs) approximately 22 nucleotides long, involved in gene expression regulation at the post-transcriptional level [1]. They play a fundamental role in almost all cellular processes including proliferation, differentiation, metabolism, growth, apoptosis, among others [2]. To date, more than 39,000 miRNAs have been described in 271 different organisms. In humans, 1,984 are reported as precursors of 2,693 mature miRNAs [3]. 
Although the function of miRNAs has been greatly characterized in the intracellular environment [4], several of these molecules have also been detected in the extracellular environment in a wide variety of body fluids, such as serum, plasma, tears, urine, breast milk, bronchoalveolar lavage, cerebrospinal fluid, semen, and amniotic fluid $[5,6]$. While the source of miRNAs in body fluids and their function in the extracellular environment remains unclear, these circulating transcripts have attracted growing interest as potential, easily accessible biomarkers of physiological alterations and pathological events. Along these lines, altered patterns of circulating miRNAs (c-miRNAs) in peripheral blood have been documented in a range of pathological conditions including cancer, heart and neuropathic diseases and perinatal complications, among others [7-12]. However, the use of c-miRNAs in clinical practice remains controversial due to challenges associated with detecting reliable signatures associated with specific physiological conditions. Important methodological caveats include the relative lack of longitudinal approaches involving temporal profiling of miRNA expression throughout the physiological condition of interest, and the scarce use of time-matched physiological data derived from the exact same subjects [11, 13].

Pregnancy is an extraordinarily complex process with important morphological, metabolic, physiological, and immunological changes involving three interacting systems, the woman, the placenta and the fetus, which maintain a complex crosstalk simultaneously aimed at maintaining women homeostasis, promote fetal growth and development, as well as preparing the mother for labor and lactation [14]. Imbalance and/or dysregulation of these processes can directly affect maternal-fetal health, putting them at risk of an ample array of perinatal complications and future effects on the health of women and offspring [15]. Pregnancy care and the capability to prevent, diagnose, and treat complications during gestation are important challenges for the clinical specialists, demanding the continuous development and improvement of accessible diagnostic tools.

Physiological evolution of pregnancy in eutherians has been associated with changes in miRNA profiles in several models [16]. In humans for instance, expression profiles of c-miRNAs clusters encoded on chromosome 14 (C14MC) and primate-specific chromosome 19 (C19MC) have been identified in the blood of pregnant women [17] and some of them have been described as footprints of maternal imprinting, as well as trophoblast and placental function $[18,19]$. Circulating expression imbalances in C14MC and C19MC members have been reported in preeclampsia, preterm delivery, or intrauterine growth retardation, suggesting some of them as potential biomarkers of these perinatal complications [20-22]. However, out of a limited set of c-miRNAs associated with placental expression and perinatal pathologies, the extent to which global profiles of circulating miRNAs in pregnant women actually reflect specific aspects related to the normal course of pregnancy remains unknown.

In the present study, using next generation sequencing-mediated profiling of peripheral blood miRNAs at four defined stages in a cohort of healthy pregnant women, in addition to non-pregnant controls in combination with detailed clinical data derived from the exact same subjects, we investigate individual longitudinal temporal changes in c-miRNA signatures, and their association with distinct aspects of normal pregnancy. Our results demonstrate the existence of temporal changes of miRNA signatures 
associated with distinct aspects of pregnancy, including correlates of placental function, fetal gender, and fetal growth as well as an early lactation-related signature; strongly suggesting the potential of peripheral miRNAs as biomarkers of healthy pregnancy.

\section{Methods}

\section{Study design}

We analyzed samples from a prospective cohort of pregnant women conducted in Mexico City, now known as "CDMX Perinatal Cohort", part of a wider study program aimed at improving conditions during the first 1,000 days of life [23].

From November 2017 pregnant women who resided in Alvaro Obregón County in the west side of metropolitan Mexico City were recruited at Dr. Enrique Cabrera General Hospital, part of the Mexico City government's public hospital network. Human subjects' approval for the study was obtained from the Instituto Nacional de Medicina Genómica Institutional Review Board (register 07/2018/I) and the Ethics in Human Subjects and Research Committees of Mexico City's Secretaría de Salud (register number: 210/010/31/17).

Inclusion criteria for women entering the cohort were: 1 ) less than 16 weeks of gestation; 2 ) reliable recall of last menstruation; 3) agreement to prenatal visits every 4-6 weeks throughout their current pregnancy; and 4) written consent for their inclusion in the study. Exclusion criteria were: 1) previous presence of any medical or obstetric complication during the current pregnancy; and 2) presence of multiple fetuses.

After screening for eligibility and written informed consent, given at the first visit or at health clinics during recruitment, women were seen every 4 to 6 weeks over the course of their pregnancies. Information on pregnancy evolution, maternal nutritional status, and fetal health, as well as biological samples were collected at each visit by a dedicated team composed of certified medical specialists and nutritionists with standardized training.

For this study, 32 plasma samples collected at early, middle, late pregnancy and after birth from eight women (PW) were selected based on the following inclusion criteria: 1) completing entire follow-up with at least three visits during pregnancy and one after birth;2) without medical or obstetrical complications during pregnancy; 3 ) delivering after 38 weeks of gestation with spontaneous labor.

Plasma samples from selected cases were grouped according to gestational age: 7.4-13.6 weeks of gestation (1T), 19.5-25.1 (2T) and 32.4-35.2 weeks of gestation (3T), and after birth (AB). In addition, plasma blood of ten healthy age-matched non-pregnant women were collected as a control group. Clinical data was collected from the associated medical records including maternal age, weight, height, weight gain during pregnancy, blood pressure, obstetric history, type of delivery, and fetal ultrasonographic measurements (gestational age, head and abdominal circumferences, femur length, and fetal weight). 


\section{Plasma samples processing and RNA isolation}

Ten $\mathrm{mL}$ of peripheral blood from each subject (in fasting conditions) were collected in tubes containing EDTA and centrifuged at 1,500 x rpm for 10 minutes. Plasma was transferred into fresh RNAse-free 1.5 $\mathrm{mL}$ tubes and stored at $-75^{\circ} \mathrm{C}$ until used.

Prior to total RNA isolation, $2 \mathrm{~mL}$ of plasma were centrifuged at $12,000 \mathrm{xg}$ for 10 minutes at $4^{\circ} \mathrm{C}$ and transferred to new tubes, to fully eliminate cellular debris. Total RNA was isolated following the manufacturer's procedure. Briefly, TRI Reagent Solution (Ambion, Thermo Fisher Scientific, USA) was added to plasma samples, in a 3:1 sample volume ratio, mixed and incubated for 5 minutes at room temperature. Then chloroform ( $0.2 \mathrm{~mL}$ per $\mathrm{mL}$ of TRI Reagent) was added and thoroughly mixed. Once centrifuged at $12,000 \mathrm{~g} \mathrm{x} \mathrm{g}$ for 10 minutes at $4^{\circ} \mathrm{C}$, the aqueous phase was transferred to fresh tubes, glycogen (Roche) and isopropanol ( $0.5 \mathrm{~mL}$ per $\mathrm{mL}$ of TRI Reagent) were added, mixed, and incubated overnight at $-75^{\circ} \mathrm{C}$. After incubation samples were centrifuged at $12,000 \mathrm{xg}$ for 15 minutes at $4^{\circ} \mathrm{C}$. RNA pellet was resuspended in $75 \%$ ethanol and centrifuged at $7,500 \times \mathrm{g}$ at $4^{\circ} \mathrm{C}$ for 5 minutes. Finally, washed RNA pellet was resuspended in $10 \mu \mathrm{L}$ of RNase-free water and stored at $-75^{\circ} \mathrm{C}$.

\section{Small RNA sequencing and miRNA profiling}

Library construction for small RNA sequencing analysis was performed with the NextFLEX small RNASeq kit v3 (Bioo Scientific, USA), bands in an acrylamide gel, ranging from 50 to 150 bp were processed. Libraries were sequenced for 150 cycles, single-end, on an Illumina NextSeq 500 at the Institute's Sequencing Core Unit.

For data analysis, fastq files were downloaded from BaseSpace Hub (Illumina). Preliminary quality control analysis of these files was performed with FastQC v0.11.4 [24], then cutadapt v1.17 [25] was used to trim $3^{\prime}$ adapters and filter sequences by phred quality score of 30, length -minimum of $18 \mathrm{bp}$ and maximum of $100 \mathrm{bp}$, and only those sequences where the $3^{\prime}$ adapter was trimmed were kept for further analysis. After this, a second quality control analysis was performed on filtered sequences with FastQC. Sequences were aligned to the human genome (Hg38p12) with BWA v0.7.12 [26] and miRNA profile was determined using miRDeep2 [27] and miRBase v.22 [3] as annotated references of mature miRNAs. Finally, reads were tallied to generate total counts for each miRNA.

\section{Data normalization and differential expression analysis}

An expression database was compiled with the total read counts from each group (pregnant and nonpregnant women) and stage (first, second and third month of pregnancy as well as after birth). A cutoff of $\geq 5$ read counts was applied before quantile normalization using the R Bioconductor v3.11 preprossCode package v1.50.0 [28]. Normalized read counts were analyzed by multidimensional scaling (MDS) to assess the similarity of global expression patterns between groups. miRNA expression levels were quantified carrying out the optimal discovery procedure and generalized likelihood ratio tests by $\mathrm{R}$ Bioconductor EdgeR package v3.30.3 [29]. 


\section{Processing of externally sourced expression data}

Available miRNA-seq expression data in placenta, umbilical cord plasma, amniotic fluid, and early breast milk (48h post-partum) were obtained from the NCBI Gene Expression Omnibus (GEO) (accession numbers: GSE114349, GSE112343, GSE107524) [6, 30, 31]. Expression values were quantile normalized as described above and sorted by average expression level to identify the miRNAs comprising the top quartile most prominently expressed in each source tissue or fluid. The identified sets of miRNAs, in addition to miRNAs belonging to the C19MC and C14MC [22], were then extracted from our circulating miRNA expression data and Wilcoxon signed-rank tests were used to asses significant collective changes, of these sets, between each pregnancy stages and non-pregnant women.

\section{Programming and statistical software}

Large-scale data handling and calculations, coding, numerical simulations and statistical analyses were carried out in R.

\section{Results}

\section{Characteristics of participants}

Clinical characteristics of pregnant women and non-pregnant control groups in this study are listed in Table 1. Pregnant women had healthy pregnancies ending at term without complications, normal ranges for pregnancy weight gain, blood pressure, and metabolic parameters according to gestational age. Indicators of fetal growth showed normal ranges, according to the World Health Organization fetal growth charts [32]. Non-pregnant women were paired for age and body mass index, showing a normal state of health. 
Table 1

Pregnancy and non-pregnancy characteristics of healthy women.

\begin{tabular}{|c|c|c|c|c|c|}
\hline \multirow[t]{2}{*}{ Features } & \multirow[t]{2}{*}{ NPW $(n=10)$} & \multicolumn{4}{|l|}{$P W(n=8)$} \\
\hline & & $\begin{array}{l}1 \mathrm{~T} 7.4- \\
13.6 \mathrm{w}\end{array}$ & $\begin{array}{l}2 \mathrm{~T} 19.5- \\
25.1 \mathrm{w}\end{array}$ & $\begin{array}{l}\text { 3Т } 32.4-35.2 \\
\text { w }\end{array}$ & $\begin{array}{l}A B \text { 3.4- } \\
10.7 \mathrm{w}\end{array}$ \\
\hline \multicolumn{6}{|c|}{ Clinical measurements } \\
\hline Age (years) & $26(22,37)$ & \multicolumn{4}{|l|}{$24(21,34)$} \\
\hline Height (cm) & $159(153,163)$ & \multicolumn{4}{|l|}{$\begin{array}{l}153(144, \\
163)\end{array}$} \\
\hline Weight (g) & $58(52,64)$ & $62(46,90)$ & $63(51,92)$ & $67(58,93)$ & $61(49,90)$ \\
\hline $\mathrm{SBP}(\mathrm{mmHg})$ & $110(107,120)$ & $\begin{array}{l}110(100 \\
121)\end{array}$ & $\begin{array}{l}102(96 \\
128)\end{array}$ & $104(99,118)$ & $\begin{array}{l}104(94 \\
113)\end{array}$ \\
\hline $\mathrm{DBP}(\mathrm{mmHg})$ & $77(70,85)$ & $70(64,85)$ & $60(55,70)$ & $67(55,75)$ & $63(60,65)$ \\
\hline MAP $(\mathrm{mmHg})$ & $90(83,94)$ & $\begin{array}{l}68 *(35 \\
84)\end{array}$ & $75(69,88)$ & $79(70,89)$ & $76(71,81)$ \\
\hline \multicolumn{2}{|l|}{ WG (kg) } & $\begin{array}{l}0.1^{\mathrm{a}}(-1.7 \\
7.4)\end{array}$ & $2.1(0.4,5.6)$ & $4.3(1.3,7.2)$ & $\begin{array}{l}-4.1^{\mathrm{a}}(-9.4 \\
-1)\end{array}$ \\
\hline \multirow{2}{*}{$\begin{array}{l}\text { Obstetric } \\
\text { history }\end{array}$} & Gravidity & \multicolumn{2}{|c|}{$3(1,4)$} & & \\
\hline & Parity & \multicolumn{2}{|c|}{$2(1,4)$} & & \\
\hline \multirow{2}{*}{$\begin{array}{l}\text { Type of } \\
\text { delivery }\end{array}$} & Vaginal & \multicolumn{2}{|c|}{$7(87.5)$} & & \\
\hline & Cesarean & \multicolumn{2}{|c|}{$1(12.5)$} & & \\
\hline \multicolumn{6}{|c|}{ Fetal ultrasonographic measurements } \\
\hline \multicolumn{2}{|l|}{ FW (g) } & $98(58,99)$ & $\begin{array}{l}431(308, \\
751)\end{array}$ & $\begin{array}{l}2151^{\mathrm{a}}(2004 \\
2496)\end{array}$ & \\
\hline \multicolumn{2}{|l|}{$\mathrm{FL}(\mathrm{cm})$} & $\begin{array}{l}1^{\mathrm{a}}(0.7 \\
1.4)\end{array}$ & $4^{\mathrm{a}}(2.9,4.6)$ & $6^{\mathrm{b}}(5.8,6.8)$ & \\
\hline \multicolumn{2}{|l|}{$\mathrm{CC}(\mathrm{cm})$} & $\begin{array}{l}10^{\mathrm{a}}(6.9 \\
11)\end{array}$ & $\begin{array}{l}19^{\mathrm{a}}(16.4 \\
22.3)\end{array}$ & $30^{\mathrm{b}}(28.3,32.4)$ & \\
\hline \multicolumn{2}{|l|}{$\mathrm{AC}(\mathrm{cm})$} & $8^{a}(6,8.6)$ & $\begin{array}{l}17^{a}(14.4 \\
20.7)\end{array}$ & $30^{\mathrm{b}}(28.8,31.7)$ & \\
\hline \multirow[t]{2}{*}{ Sex } & Girl & \multicolumn{4}{|l|}{$5(62.5)$} \\
\hline & Boy & \multicolumn{4}{|l|}{3 (37.5) } \\
\hline
\end{tabular}

Data are the median (interquartile range) or $\mathrm{n}(\%)$. Mann Whitney test were performed to analyze statistical difference between NPW and PW at 1T (asterisk, *) and Kruskal Wallis test were used to 
analyze differences between stages of pregnancy (different letters). NPW: non-pregnant women, PW: pregnant woman, 1T: 1st trimester of pregnancy, $2 \mathrm{~T}$ : $2 \mathrm{nd}$ trimester, $3 \mathrm{~T}$ : 3rd trimester, $\mathrm{AB}$ : after birth, w: weeks, SBP: systolic blood pressure, DBP: diastolic blood pressure, MAP: mean arterial pressure, WG: weight gain, FW: fetal weight, FL: femur length, CC: cephalic circumference, AC: abdominal circumference.

\section{c-miRNA expression profiles in pregnant and non-pregnant women}

Plasma-derived small RNA sequencing was carried out for each subject at each pregnancy stage using an Illumina Next sequencing platform. After quality control and genome annotation, a total of 1,449 mature microRNAs were detected overall in the studied samples. Raw read count data (for $\geq 5$ reads per sample) for each individual sample and corresponding metadata is available. To determine the level of similarity or difference in the global c-miRNA expression profiles in plasma between pregnant and nonpregnant women, as well as between individuals and pregnancy stages $(1 T, 2 T, 3 T$, and $A B)$, we conducted a multidimensional scaling analysis after quantile normalization of the expression data. As shown in Fig. 1A, minor gross global differences in expression profiles were observed between individuals or stages, strongly suggesting small differences overall in the collective expression of c-miRNAs across individuals and stages.

To identify potential expression differences at the level of individual c-miRNAs, we carried out a differential expression analysis across all 1,449 c-miRNAs detected in the maternal plasma. We compared the expression between each of the four pregnancy stages of study and corresponding expression in non-pregnant controls. As shown in Fig. 1B, an increasing number of statistically significant (FDR < 0.05) differentially expressed c-miRNAs between pregnant and non-pregnant women were detected for each successive trimester of pregnancy, from $10 \mathrm{c}$-miRNAs in the 1T, through to 43 in the $3 \mathrm{~T}$ followed by a reduction after birth (AB). By examining the overlap between subsets of differentially expressed c-miRNAs at each period (Fig. 1C), we found a statistically significant overlap throughout pregnancy (Fig. 1C, Fisher exact test $p$ value $0.0014,0.0004$ and 0.0003 , respectively) indicating a single core of c-miRNAs displaying increasing differences in expression as pregnancy progresses, with the number of differentially expressed c-miRNAs virtually vanishing after birth. When analyzing the changes in expression of the pulled set of differentially expressed transcripts across all four pregnancy stages ( $\mathrm{n}$ = 46), we found a rapid increase in collective expression, from a 16 fold increase in median expression during the first trimester $\left(\log _{2} \mathrm{FC}=4\right)$, through to a 250 fold increase during the third trimester $\left(\log _{2} \mathrm{FC}=\right.$ 8). Maximal increases ranged from 250 fold during the first trimester to over 2000 fold change during the third trimester, with all differences drastically dropping after birth (Fig. 1D and Table 2).

Figure 1. Global comparison of c-miRNA expression profile throughout pregnancy. A) Global similarity in normalized c-miRNA expression profiles between both individual pregnant and non-pregnant women, as well as across stages, being revealed by multidimensional scaling analysis. B) Bar chart indicating the total number of differentially expressed c-miRNAs (grey), overexpressed (black) and sub expressed 
(white) when comparing pregnant women at each indicated period with non-pregnant control subjects. C) Venn diagram illustrating overlaps of differentially expressed c-miRNAs across all four stages. Asterisks indicate significance in overlap (Fisher exact test $p<0.005$ ). D) Boxplot charts show increasing expression level among differentially expressed c-miRNAs during $1 \mathrm{~T}, 2 \mathrm{~T}, 3 \mathrm{~T}$, and a subsequent drop at $\mathrm{AB}$. (1T: first trimester of pregnancy, 2T: second, 3T: third, AB: after birth). 
Table 2

Differential expression of c-miRNAs between pregnant and non-pregnant women.

\section{$\log _{2} \mathrm{FC}$}

$\log _{2} \mathrm{FC}$

\begin{tabular}{|c|c|c|c|c|c|c|c|c|c|}
\hline miRNA-ID & $1 T$ & $2 T$ & 3T & $A B$ & miRNA-ID & $1 T$ & $2 T$ & $3 T$ & $A B$ \\
\hline $\begin{array}{l}\text { hsa-miR- } \\
122-5 p\end{array}$ & $2.19 *$ & $3.04 *$ & $2.56^{\star}$ & $3.13^{*}$ & $\begin{array}{l}\text { hsa-miR- } \\
501-3 p\end{array}$ & 0.86 & 1.36 & $1.55^{\star}$ & 0.74 \\
\hline $\begin{array}{l}\text { hsa-miR- } \\
518 \mathrm{e}-5 p\end{array}$ & 9.50 * & $9.50 *$ & $12.28^{*}$ & 0.00 & $\begin{array}{l}\text { hsa-miR- } \\
524-5 p\end{array}$ & 6.80 & 6.06 & $10.12^{\star}$ & 0.00 \\
\hline $\begin{array}{l}\text { hsa-miR- } \\
519 a-5 p\end{array}$ & $9.50 *$ & $9.50 *$ & $12.28^{*}$ & 0.00 & $\begin{array}{l}\text { hsa-miR- } \\
\text { 525-5p }\end{array}$ & 7.00 & 0.00 & $9.99 *$ & 0.00 \\
\hline $\begin{array}{l}\text { hsa-miR- } \\
519 b-5 p\end{array}$ & $9.50 *$ & $9.50 *$ & $12.28^{*}$ & 0.00 & $\begin{array}{l}\text { hsa-miR- } \\
518 f-5 p\end{array}$ & 4.41 & 0.00 & $9.44^{\star}$ & 0.00 \\
\hline $\begin{array}{l}\text { hsa-miR- } \\
519 c-5 p\end{array}$ & $9.50 *$ & $9.50 *$ & $12.28^{*}$ & 0.00 & $\begin{array}{l}\text { hsa-miR- } \\
518 d-5 p\end{array}$ & 4.41 & 0.00 & $9.44 *$ & 0.00 \\
\hline $\begin{array}{l}\text { hsa-miR- } \\
522-5 p\end{array}$ & $9.50 *$ & $9.50 *$ & $12.28^{\star}$ & 0.00 & $\begin{array}{l}\text { hsa-miR- } \\
526 a-5 p\end{array}$ & 4.41 & 0.00 & $9.44^{*}$ & 0.00 \\
\hline $\begin{array}{l}\text { hsa-miR- } \\
523-5 p\end{array}$ & $9.50 *$ & $9.50 *$ & $12.28^{*}$ & 0.00 & $\begin{array}{l}\text { hsa-miR- } \\
520 c-5 p\end{array}$ & 4.41 & 0.00 & $9.44^{*}$ & 0.00 \\
\hline $\begin{array}{l}\text { hsa-miR- } \\
1323\end{array}$ & $7.22 *$ & $7.93^{\star}$ & $10.48^{\star}$ & 1.07 & $\begin{array}{l}\text { hsa-miR- } \\
518 \mathrm{~b}\end{array}$ & 7.65 & 6.49 & $9.21 *$ & 0.00 \\
\hline $\begin{array}{l}\text { hsa-miR- } \\
519 a-2-5 p\end{array}$ & $8.65^{\star}$ & 6.53 & $9.88 *$ & 0.00 & $\begin{array}{l}\text { hsa-miR- } \\
498-5 p\end{array}$ & 6.77 & 5.90 & $8.92^{\star}$ & 0.00 \\
\hline $\begin{array}{l}\text { hsa-miR- } \\
\text { 520b-5p }\end{array}$ & $8.65^{\star}$ & 6.53 & $9.88 *$ & 0.00 & $\begin{array}{l}\text { hsa-miR- } \\
1283\end{array}$ & 3.36 & 0.00 & $8.13^{\star}$ & 0.00 \\
\hline $\begin{array}{l}\text { hsa-miR- } \\
516 b-5 p\end{array}$ & 7.12 & $9.16^{\star}$ & $11.09 *$ & 0.00 & $\begin{array}{l}\text { hsa-miR- } \\
519 d-5 p\end{array}$ & 2.85 & 0.00 & $8.38 *$ & 0.00 \\
\hline $\begin{array}{l}\text { hsa-let-7b- } \\
3 p\end{array}$ & 1.35 & $1.25^{\star}$ & $2.51^{\star}$ & 0.71 & $\begin{array}{l}\text { hsa-miR- } \\
520 d-3 p\end{array}$ & 4.12 & 0.00 & $8.09 *$ & 0.00 \\
\hline $\begin{array}{l}\text { hsa-miR- } \\
516 a-5 p\end{array}$ & 7.61 & 8.11 & $11.00 *$ & 2.78 & $\begin{array}{l}\text { hsa-miR- } \\
520 a-5 p\end{array}$ & 0.00 & 0.00 & $7.86^{\star}$ & 0.00 \\
\hline $\begin{array}{l}\text { hsa-miR- } \\
4748\end{array}$ & 4.19 & 6.12 & $9.00 *$ & 2.22 & $\begin{array}{l}\text { hsa-miR- } \\
4485-3 p\end{array}$ & 6.61 & 6.18 & $7.64^{*}$ & 2.42 \\
\hline $\begin{array}{l}\text { hsa-miR- } \\
522-3 p\end{array}$ & 5.19 & 7.67 & $9.12^{*}$ & 0.00 & $\begin{array}{l}\text { hsa-miR- } \\
518 \mathrm{e}-3 p\end{array}$ & 3.25 & 0.00 & $7.64^{\star}$ & 0.00 \\
\hline $\begin{array}{l}\text { hsa-miR- } \\
518 a-3 p\end{array}$ & 3.59 & 4.61 & $8.42^{\star}$ & 0.00 & $\begin{array}{l}\text { hsa-miR- } \\
518 f-3 p\end{array}$ & 4.06 & 0.00 & $7.41^{*}$ & 0.00 \\
\hline $\begin{array}{l}\text { hsa-miR- } \\
526 b-5 p\end{array}$ & 4.48 & 6.14 & $8.28 *$ & 3.14 & $\begin{array}{l}\text { hsa-miR- } \\
133 a-3 p\end{array}$ & -3.57 & -2.96 & $-4.02^{\star}$ & -1.16 \\
\hline
\end{tabular}




\begin{tabular}{|c|c|c|c|c|c|c|c|c|c|}
\hline \multicolumn{6}{|c|}{$\log _{2} F C$} & \multicolumn{4}{|c|}{$\log _{2} \mathrm{FC}$} \\
\hline $\begin{array}{l}\text { hsa-miR- } \\
518 c-3 p\end{array}$ & 0.00 & 3.30 & 7.99* & 0.00 & $\begin{array}{l}\text { hsa-miR- } \\
\text { 145-5p }\end{array}$ & -1.57 & -1.40 & $-1.60 *$ & -0.67 \\
\hline $\begin{array}{l}\text { hsa-miR- } \\
517 a-3 p\end{array}$ & 3.76 & 4.42 & $6.59 *$ & 0.40 & $\begin{array}{l}\text { hsa-miR- } \\
181 d-5 p\end{array}$ & -1.14 & -0.73 & $-1.83^{\star}$ & -0.68 \\
\hline $\begin{array}{l}\text { hsa-miR- } \\
517 b-3 p\end{array}$ & 3.76 & 4.42 & $6.59 *$ & 0.40 & $\begin{array}{l}\text { hsa-miR- } \\
423-5 p\end{array}$ & 1.63 & 1.65 & $1.52^{\star}$ & 0.69 \\
\hline $\begin{array}{l}\text { hsa-miR- } \\
483-5 p\end{array}$ & 2.68 & 2.82 & $4.41^{\star}$ & 3.77 & $\begin{array}{l}\text { hsa-miR- } \\
328-5 p\end{array}$ & 6.75 & $9.98 *$ & 0.00 & 3.91 \\
\hline $\begin{array}{l}\text { hsa-let-7d- } \\
3 p\end{array}$ & 1.62 & 1.69 & $1.88 *$ & 0.87 & $\begin{array}{l}\text { hsa-miR- } \\
\text { 548ag }\end{array}$ & 0.26 & $-8.76^{\star}$ & 0.01 & -1.39 \\
\hline $\begin{array}{l}\text { hsa-miR- } \\
10 \mathrm{~b}-5 \mathrm{p}\end{array}$ & 1.30 & 1.06 & $1.89 *$ & 1.54 & $\begin{array}{l}\text { hsa-miR- } \\
6750-5 p\end{array}$ & 0.00 & 4.69 & 0.00 & $10.44^{*}$ \\
\hline
\end{tabular}

Differential expression analysis between fold changes of each stage of pregnancy and after birth, and non-pregnant women using R package "EDGAR" (FDR < 0.05). 1T: first trimester of pregnancy, 2T: second, 3T: third, $A B$ : after birth.

These results show that, while global expression profiles of c-miRNAs in pregnant women show moderate collective differences, relative to non-pregnant women, there is a gradual and increase in differential expression in a small population as pregnancy progresses, followed by a pronounced drop at the immediate post pregnancy period. These results also suggest that changes in c-miRNAs associated with pregnancy could be restricted to distinct subpopulations.

\section{c-miRNA subsets associated with tissue compartments of pregnancy}

In order to assess if changes in circulating subpopulations of c-miRNAs in pregnant women reflect events specifically associated to pregnancy, we examined the collective expression of miRNA belonging to the C14MC and C19MC families, known for their prominent involvement in trophoblast differentiation and function [22, 33]. For this objective, we carried out a paired comparison (Wilcoxon signed Rank test) for these signatures between pregnant and non-pregnant women, for each studied period. As shown in Fig. 2A and 2B both families display significant expression changes in pregnant women, relative to nonpregnant controls, with their collective expression returning to control levels after birth. Interestingly, the observed direction of differential expression was different for each of these families, with the C14MC family collectively displaying a significantly down-regulated expression during pregnancy, mainly during the second trimester. The C19MC family was found up-regulated during pregnancy, especially in the third trimester and returning to control levels after birth. Together these results show that c-miRNA families display a significant change in expression in plasma of pregnant women, returning to control (nonpregnancy) levels within three months after birth. 
Figure 2. Trophoblast-associated miRNA families expressed in the plasma of pregnant women. A) Bar chart showing median difference in the expression of C14MC $(n=84)$ between pregnant and nonpregnant samples at each indicated stage during and after pregnancy. B) Bar chart showing median difference in expression of the C19MC ( $=54)$ between pregnant and non-pregnant samples at each indicated stage during and after pregnancy. $\mathrm{P}$ values for each comparison (paired Wilcoxon paired test) are indicated for each individual comparison.

Using a complementary approach, we obtained miRNA expression data from existing literature for normal placenta [30], amniotic fluid, umbilical cord plasma [6], and breast milk [31], to define indicative miRNA subpopulations associated, but not necessarily, specific to each of these tissues of fluids. Theses compartment-associated subsets were defined as the topo $25 \%$ miRNAs most prominently expressed in each fluid or tissue also present among the 1,449 c-miRNAs detected in this study (For details see Additional file 1).

Accordingly, we compared the expression of four different sets of $362 \mathrm{c}$-miRNAs in pregnant women corresponding to the reported top quartile expression in placenta, umbilical fluid, umbilical cord plasma, and breast milk relative to their expression in non-pregnant women. As shown in Fig. 3, all four signatures display highly significant collective changes in expression in maternal plasma, relative to non-pregnant women at each stage of pregnancy, with their collective expression dropping to minimal differences after birth. In every case, the change in median expression revealed a down regulation of theses miRNA subsets in maternal plasma during pregnancy relative to non-pregnant controls.

Figure 3. Down regulation of c-miRNAs most highly expressed in placenta, amniotic fluid, umbilical cord plasma, and breast milk in plasma of pregnant women at four different stages during and after pregnancy. The expression of the top $25 \%$ most highly expressed miRNAs in each of the indicated tissues and fluids was measured in maternal peripheral plasma and compared against that of non pregnant women by paired Wilcoxon test. A) Bar chart showing the median difference in expression of c-miRNAs most highly expressed in placenta $(n=362)$ at each indicated stage. B) Bar chart showing the median difference in expression of c-miRNAs most highly expressed in amniotic fluid $(n=362)$. C) Bar chart showing median difference in expression of c-miRNAs most highly expressed in umbilical cord plasma ( $\mathrm{n}$ = 362). D) Bar chart sowing mean difference in expression of c-miRNAs most highly expressed in breast milk $(n=362)$ at each indicated stage. $1 T, 2 T, 3 T$ and $A B$ : first trimester, second trimester, third trimester and after birth, respectively. $P$ values (paired Wilcoxon signed rank test) are indicated under each bar.

We confirmed that each of the four signatures consist of distinct subpopulations of c-miRNAs with little overlap between them, and that each signature is associated specifically to the fluid or tissues where it came from (See Additional file 2). Taken together, these results demonstrate that subpopulations of cmiRNAs associated with pregnancy-specific tissues show significant alterations in their collective level of expression in maternal plasma throughout all pregnancy stages, returning to near non-pregnant levels after birth. So far, these results demonstrate that certain pregnancy specific events occurring in specific 
compartments, including breast milk, are reflected by changes in distinct subpopulations of maternal cmiRNAs during pregnancy.

\section{c-miRNA subsets associated with fetal sex signal}

To further probe the kind of information that could potentially be found embedded in the global profile of maternal c-miRNAs during pregnancy, we asked if more specific fetal-related variables could be detected. One such trait would be fetal sex. In order to elucidate if fetal sex-specific information could be present in c-miRNAs at any stage during pregnancy, we conducted a differential expression analysis across all 1,449 miRNAs detected in maternal circulation, comparing to women who eventually gave birth to female or male babies. Of all the women included in this study, five gave birth to a baby girl and three to a baby boy. This analysis resulted in no individual c-miRNAs displaying statistically significant expression changes between these two groups of women. While this result suggests that no individual c-miRNAs carries information on fetal sex during pregnancy, it could still be possible that fetal sex-specific information could be evidenced at the population level.

We tested this notion by obtaining the total number of c-miRNAs increasing their expression in women giving birth to female babies relative to women giving birth to male babies and assessing the bias in the number of up or down regulated transcripts relative to chance expectations. As shown in Fig. 4, the observed number of up (red arrow, 911 for $1 \mathrm{~T}, 978$ for $2 \mathrm{~T}, 765$ for $3 \mathrm{~T}$, and 830 for $A B$ ) and down (blue arrow, 538 for $1 \mathrm{~T}, 471$ for $2 \mathrm{~T}, 422$ for $3 \mathrm{~T}$, and 619 for $\mathrm{AB}$ ) regulated miRNAs for each indicated trimester, displayed a highly significant bias when compared with the expected distribution of both up or down regulated miRNAs, estimated using 10,000 randomizations of expression values per c-miRNA across samples for each separate time period. This strong bias was apparent right from the first trimester only becoming weaker, but still significant, after birth. This result demonstrates variations in the collective expression of distinct subsets of maternal c-miRNAs as a function of fetal sex, and that these variations can be found at each trimester during pregnancy as well as after birth.

Figure 4. Biased expression in maternal c-miRNAs associated to fetal sex during pregnancy. Differences in expression in c-miRNAs between women giving birth to female babies relative to women giving birth to male babies were calculated and the total number of transcripts increasing their expression was obtained for each pregnancy period regardless of statistical significance at the level of individual miRNAs. A-D) Observed number of up (red arrow) and down (blue arrow) regulated miRNAs for each indicated trimester compared with the expected distribution of both up or down regulated miRNAs estimated using 10,000 randomizations of expression values per gene across samples for each separate time period. Arrows located outside the expected distribution indicate a $\mathrm{p}$ value $<0.0001$.

\section{c-miRNAs signature associated with fetal growth}

We next looked for a potential association between maternal c-miRNAs and fetal growth, as an additional continuous measure of normal pregnancy progression. To this end, we used indicators of fetal growth (ultrasonography-estimated fetal weight and femur length) obtained from the exact same women at each trimester and calculated the Spearman correlation between expression level of each individual c-miRNA 
and both indicators. We first removed all c-miRNAs with no detectable expression in $50 \%$ or more of the samples (mostly lowly expressed transcripts), resulting in a total $588 \mathrm{c}$-miRNAs with detectable expression in at least $50 \%$ of the samples. We then selected the top and bottom tenth most positively and negatively correlated c-miRNAs, respectively. For each of these two top and bottom subpopulations $(\mathrm{n}=$ 60 , each) we created a series of gradually larger signatures by successively adding one by one each cmiRNA starting from the one with the highest absolute correlation value, and summarized the collective expression of the resulting partial signature through their associated eigengene (a vector capturing the maximum variance of a set of genes [34]).

For each successive partial signature, we calculated the Spearman correlation against fetal weight and fetal length to identify the subset of c-miRNAs revealing the strongest collective association with fetal growth. As shown in Fig. 5, the correlation of each successive signature's expression (summarized by their associated eigengene) and fetal growth shows a peak at the top 56 (Fig. 5A) and 43 (Fig. 5C), respectively most positively correlated c-miRNAs, pointing to these subsets as the one displaying the highest collective association with both fetal growth indicators $(R=0.7, p<0.01)$.

To look at how the strength of this signature compares with what could be expected by chance, we randomized the expression data before repeating the same procedure we followed to detect the above signature and found that the resulting randomized signatures failed to reach the effect sizes derived from the actual data (dotted red and blue lines in the short insight of Fig. 5). To determine the statistical significance of this difference, we conducted 10,000 independent randomizations of expression data to extract, in every case the optimal signature, and determined the probability of obtaining a similar signature (of the same size and strength). As shown in the inset of Fig. 5A and $C$, the observed real signatures are highly unlikely to occur by chance $(p<0.05)$.

Figure 5. c-miRNA signatures of fetal growth throughout pregnancy. Spearman correlations between fetal growth indicators and transcript abundance were calculated for every miRNA present in maternal circulation across pregnancy samples. A sequence of partial signatures was created by successively adding each c-miRNA in descending order of correlation with fetal weight and fetal length, starting from the topmost highly correlated transcript. Expression of each successive signature was summarized by their associated eigengene and correlated with both fetal growth indicators to quantify its strength of association. Left column charts show absolute Spearman correlation with fetal weight (A) and femur length (C) for each successive signature as additional c-miRNAs added in descending order of correlation starting from the topmost highly correlated transcript (red line). Right column charts show the strength of association with fetal weight (B) and femur length (D) for each successive signature as additional miRNAs added in ascending order of absolute correlation starting from the most negatively correlated transcript (blue line). Dashed lines in charts represent the same analysis carried out after expression was randomized for each transcript across all samples. The optimal signature is indicated by the vertical line. Insets show the probability of obtaining a similarly correlated optimal signature after 10,000 independent randomizations of the original expression data. 
We further confirmed that the association between the average expression of these c-miRNA signatures and indicators of fetal growth is not secondary to a concomitant change also taking place during pregnancy such as maternal body weight (Fig. 6). These results demonstrate the existence of a subpopulation of miRNAs significantly and specifically associated with fetal growth present in maternal circulation during normal pregnancy.

\section{Figure 6. Maternal c-miRNA fetal growth signatures are not associated to maternal changes in body} weight. Optimal signatures of fetal growth (fetal weight $(n=56)$ and femur length $(n=43))$ were summarized by its mean expression across samples and correlated with either both indicators of fetal growth or maternal body weight data obtained from the exact same women. Scatter graphs in the left column display the association between fetal weight (A) or femur length (C) and the mean expression of the identified optimal signature. Scatter graphs in the right column display the association between maternal body weight and the mean expression of the optimal signature identified in Fig. 5A and C. Spearman Correlation rho and $p$ values for each comparison are indicated.

\section{Discussion}

In this study, we conduct a large-scale profiling of maternal circulating miRNAs at four stages during and after healthy pregnancies. By comparing with the corresponding expression patterns in healthy nonpregnant women, our findings suggest the existence of subtle variations in miRNA profiles involving distinct but small subpopulations of transcripts potentially associated to specific pregnancy-related tissues and fluids at all stages during gestation (from the first through to third trimester) and after birth.

We identify 1,449 miRNAs in maternal circulation, which represents one of the largest sets of mature miRNAs reported in circulation [6], particularly during pregnancy [35]. While no gross differences in the global profiles were evident neither between pregnant and non-pregnant women, nor between pregnancy stages, direct differential expression analysis revealed significant differences in a relatively small number of individual miRNAs, many of which have also been described in other studies of plasma miRNAs during pregnancy [36, 37].

Limited changes in miRNA expression have indeed been reported in a variety of body fluids in numerous conditions [5,6]. Based on these observations, and our own findings in this study, we hypothesized that changes in c-miRNAs during pregnancy, if any, are more likely to be found at the level of subtle collective changes in defined miRNA subpopulations.

Along this line, we started by looking at subsets of miRNAs known to be associated with specific events of pregnancy. In this regard, miRNAs members belonging to the C14MC and C19MC families have attracted considerable attention due to their known involvement in placental function and their potential as biomarkers of placental health and embryo development [13, 22, 38-41]. Our finding of highly significant changes in the collective expression of these two signatures during all three stages of pregnancy, but not after birth, further supports the notion of a key involvement of these c-miRNA populations in healthy pregnancy. 
Significantly increased expression of C14MC and C19MC miRNAs during pregnancy, in placental tissue, have been documented [20,42], and it has been suggested that these transcripts are either actively or passively transported from the placental compartment to the maternal plasma during the natural progression of pregnancy $[22,43,44]$. This notion, however, is in direct conflict with our finding that $\mathrm{C} 14 \mathrm{MC}$ transcripts were collectively down-regulated in maternal circulation during pregnancy; a pattern of expression that opposed what has been described in placenta. This observation suggests instead that, far from being passively transported from the placental compartment, C14MC miRNAs are selectively excluded of maternal circulation and probably maintained inside of the intrauterine compartment, suggesting the existence of selective uptake mechanisms exhibited by the placenta and/or their active down regulation in maternal tissues contributing to c-miRNAs. Consistent with the notion of an active removal or down regulation of placental miRNAs, transcripts most highly expressed in placenta were also found collectively reduced throughout pregnancy but not after birth. C19MC miRNAs, on the other hand, displayed a collective increased expression during pregnancy, suggesting different mechanisms regulating the presence of $\mathrm{C} 19 \mathrm{MC}$ and $\mathrm{C} 14 \mathrm{MC}$ miRNAs in maternal plasma during the progression of normal pregnancy $[14,45]$.

Following the same approach, we examined the expression of c-miRNAs associated with other specific pregnancy related compartments, such as those present in amniotic fluid, umbilical cord serum and early breast milk which are related to the normal progression of pregnancy [14]. These reproductive compartments are involved in a wide range of adaptations to events such as implantation, maintenance, labor, lactation $[16,46]$, or even events associated with the control of inflammation and tolerance at the maternal-fetal interface $[47,48]$. Our findings demonstrated a down regulation of the collective expression of these miRNAs during all stages of pregnancy, again suggesting the possibility that the placenta or other reproductive tissues may regulate and restrict the passage of these transcripts from the intrauterine environment to the maternal circulation, or alternatively suggesting the active down regulation of these transcripts by other maternal tissues.

By looking at fluctuations in the proportions of up and down regulated miRNAs, between women giving birth to female and male babies, we document, to the best of our knowledge for the first time, highly significant biases in the expected proportions of differentially expressed c-miRNAs as a function of fetal sex at all stages during pregnancy, as well as after birth. These findings suggest possible existence of specific molecular signatures associated to fetal sex, as early as the first trimester, and opens the possibility of detecting fetal sex much earlier than it is currently possible using other methods $[49,50]$ and solely based on patterns of c-miRNAs. In this regard, no differential expression of miRNAs has been detected between male and female derived umbilical cord after a normal pregnancy. However, alterations in miRNAs between male and female fetuses have been linked to perinatal complications $[45,51]$.

Finally, by combining our longitudinal transcriptome profiling with ultrasonographic measurements of fetal growth derived from the exact same women and conducted at the exact same stages, we identify a circulating miRNA signature of fetal growth, occurring in maternal plasma, during normal pregnancy. Our randomization analyses demonstrate that the observed association between this signature and fetal 
growth was significantly higher than expected by chance, and that it was not related to changes in maternal body weight. To the best of our knowledge, this is the first report of a miRNA signature of fetal growth present in maternal plasma during the normal course of pregnancy.

The functional significance of the observed collective changes in c-miRNA subpopulations remains unclear. It is also not clear whether these changes reflect systemic maternal demands or whether they are secondary to physiological adaptations taking place in other organs or whether this reflects a new, as yet, undescribed placental function (i.e., selective take of maternal circulating transcripts). Future additional work will be needed to identify the functional roles of these changes as, in general, the functional significance of extracellular circulating miRNAs in other biofluids remains unclear.

\section{Conclusions}

In summary, our results demonstrate the existence of temporal changes of miRNA signatures and subpopulations associated to distinct aspects of gestation and pregnancy, including correlates of placental function, fetal gender, and fetal growth as well as early lactation; strongly supporting a wider potential of peripheral miRNAs as biomarkers of healthy pregnancy.

\section{Abbreviations}

c-miRNAs

circulating microRNAs

C14MC

chromosome 14 microRNA cluster

C19MC

chromosome 19 microRNA cluster

MiRNA

microRNA

\section{Declarations}

\section{Ethics approval and consent to participate}

Human subjects' approval for the study was obtained from the Instituto Nacional de Medicina Genómica Institutional Review Board (register 07/2018/I) and the Ethics in Human Subjects and Research Committees of Mexico City's Secretaría de Salud (register number: 210/010/31/17).

\section{Consent for publication}

Not applicable

\section{Availability of data and materials}


All raw and processed RNA sequencing data generated in this study have been submitted to the NCBI Gene Expression Omnibus in private status (Access number: GSE165740; secure token for reviewers: ivetsyycjjcvdij). The rest of data generated or analyzed during the study are included in this article. Further enquiries can be directed to the corresponding author.

\section{Competing interests}

The authors declare that they have no competing interests.

\section{Funding}

This work was supported by CONACyT-SEP grant A1-S-35245, Fundación Gonzalo Río Arronte grant S.633, SECTEl grant 253/2019, INMEGEN grant 07/2018/I, and FUNSALUD grant PI1000d to Dr. Felipe Vadillo-Ortega. All participants provided informed consent.

\section{Authors' contributions}

All authors agree to be accountable for all aspects of the work and read and approved the final manuscript. ECS and FVO: conceived and designed the study, reviewed data, interpretated data, supervised the study, made comments to the manuscript, and wrote it. HG: designed computational study method, analyzed computational data, supervised the study, and wrote the manuscript. ALHO and MFT: performed the genomic analysis, interpretated data, and reviewed the manuscript. KCC and SG: collected and prepared the data and reviewed the manuscript. JCCA and NCEM: followed-up perinatal care, collected clinical data, critiqued, and reviewed the manuscript. CFFJ: interpretated data, critiqued, and reviewed the manuscript. FVO: obtained the financial support.

\section{Acknowledgements}

Betzabett Morales Rodríguez, Filiberto Augusto Bautista Moreno, Haydee Miranda Ortiz, and Patricia Rosas Escobar for the technical support.

\section{References}

1. Bartel DP. MicroRNAs: genomics, biogenesis, mechanism, and function. Cell. 2004;116:281-97.

2. Saliminejad K, Khorram Khorshid HR, Soleymani Fard S, Ghaffari SH. An overview of microRNAs: Biology, functions, therapeutics, and analysis methods. J Cell Physiol. 2019;234:5451-65.

3. Kozomara A, Birgaoanu M, Griffiths-Jones S. miRBase: from microRNA sequences to function. Nucleic Acids Res. Oxford University Press; 2019;47:pp. D155-62.

4. Bartel DP. MicroRNA Target Recognition and Regulatory Functions. Cell. 2009;136:215-33.

5. El-Mogy M, Lam B, Haj-Ahmad TA, McGowan S, Yu D, Nosal L, et al. Diversity and signature of small RNA in different bodily fluids using next generation sequencing. BMC Genomics BMC Genomics. 2018;19:1-24. 
6. Godoy PM, Bhakta NR, Barczak AJ, Cakmak H, Fisher S, MacKenzie TC, et al. Large Differences in Small RNA Composition Between Human Biofluids. Cell Rep [Internet]. ElsevierCompany;; 2018;25:1346-58. Available from: https://doi.org/10.1016/j.celrep.2018.10.014.

7. Ghai V, Baxter D, Wu X, Kim TK, Kuusisto J, Laakso M, et al. Circulating RNAs as predictive markers for the progression of type 2 diabetes. J Cell Mol Med. 2019;23:2753-68.

8. Huang X, Yuan T, Tschannen M, Sun Z, Jacob H, Du M, et al. Characterization of human plasmaderived exosomal RNAs by deep sequencing. BMC Genom. 2013;14:1-14.

9. Li X, Zhao Z. MicroRNA biomarkers for early detection of embryonic malformations in pregnancy. J Biomol Res Ther. 2014;3:1-8.

10. Timofeeva AV, Gusar VA, Kan NE, Prozorovskaya KN, Karapetyan AO, Bayev OR, et al. Identification of potential early biomarkers of preeclampsia. Placenta. 2018;61:61-71.

11. Wang H, Peng R, Wang J, Qin Z, Xue L. Circulating microRNAs as potential cancer biomarkers: The advantage and disadvantage. Clin Epigenetics Clinical Epigenetics. 2018;10:1-10.

12. Akat KM, Moore-McGriff D, Morozov P, Brown M, Gogakos T, Da Rosa JC, et al. Comparative RNAsequencing analysis of myocardial and circulating small RNAs in human heart failure and their utility as biomarkers. Proc Natl Acad Sci U S A. 2014;111:11151-6.

13. Carbone IF, Conforti A, Picarelli S, Morano D, Alviggi C, Farina A. Circulating Nucleic Acids in Maternal Plasma and Serum in Pregnancy Complications: Are They Really Useful in Clinical Practice? A Systematic Review. Mol Diagnosis Ther [Internet]. Springer International Publishing; 2020;24:409-31. Available from: https://doi.org/10.1007/s40291-020-00468-5.

14. Soma-Pillay P, Nelson-Piercy C, Tolppanen H, Mebazaa A. Physiological changes in pregnancy. Cardiovasc J Afr. 2016;27:89-94.

15. Gaccioli F, Lager S, Sovio U, Charnock-Jones DS, Smith GCS. The pregnancy outcome prediction (POP) study: Investigating the relationship between serial prenatal ultrasonography, biomarkers, placental phenotype and adverse pregnancy outcomes. Placenta [Internet]. Elsevier Ltd; 2017;59:S17-25. Available from: https://doi.org/10.1016/j.placenta.2016.10.011.

16. Bidarimath $\mathrm{M}$, Khalaj K, Wessels JM, Tayade C. MicroRNAs, immune cells and pregnancy. Cell Mol Immunol. 2014;11:538-47.

17. Miura K, Miura S, Yamasaki K, Higashijima A, Kinoshita A, Yoshiura KI, et al. Identification of pregnancy-associated microRNAs in maternal plasma. Clin Chem. 2010;56:1767-71.

18. Donker RB, Mouillet JF, Chu T, Hubel CA, Stolz DB, Morelli AE, et al. The expression profile of C19MC microRNAs in primary human trophoblast cells and exosomes. Mol Hum Reprod. 2012;18:417-24.

19. Valbuena GN, Apostolidou S, Roberts R, Barnes J, Alderton W, Harper L, et al. The 14q32 maternally imprinted locus is a major source of longitudinally stable circulating microRNAs as measured by small RNA sequencing. Sci Rep [Internet]. Springer US; 2019;9:1-12. Available from: http://dx.doi.org/10.1038/s41598-019-51948-6.

20. Miura K, Higashijima A, Murakami Y, Tsukamoto O, Hasegawa Y, Abe S, et al. Circulating chromosome 19 miRNA cluster microRNAs in pregnant women with severe pre-eclampsia. J Obstet 
Gynaecol Res. 2015;41:1526-32.

21. Montenegro D, Romero R, Sung-Su K, Tarca AL, Draghici S, Kusanovic JP, et al. Expression Patterns of MicroRNAs in the Chorioamniotic Membranes: a Role for MicroRNAs in Human Pregnancy and Parturition. J Pathol [Internet]. 2009;217:113-21. Available from:

https://www.ncbi.nlm.nih.gov/pmc/articles/PMC3624763/pdf/nihms412728.pdf.

22. Morales-Prieto DM, Ospina-Prieto S, Chaiwangyen W, Schoenleben M, Markert UR. Pregnancyassociated miRNA-clusters. J Reprod Immunol [Internet]. Elsevier Ireland Ltd; 2013;97:51-61. Available from: http://dx.doi.org/10.1016/j.jri.2012.11.001.

23. Estrada-Gutiérrez G, Zambrano E, Polo-Oteyza E, Cardona-Pérez A, Vadillo-Ortega F. Intervention during the first 1000 days in Mexico. Nutr Rev. 2020;78:80-90.

24. Andrews S. FastQC: a quality control tool for high throughput sequence data. http://www.bioinformatics.babraham.ac.uk/projects/fastqc. 2010.

25. Martin M. Cutadapt removes adapter sequences from high-throughput sequencing reads. EMBnet.journal [Internet]. 2011; Available from: http://journal.embnet.org/index.php/embnetjournal/article/view/200/479.

26. Li H, Durbin R. Fast and accurate short read alignment with Burrows-Wheeler transform. Bioinformatics. 2009;25:1754-60.

27. Friedländer MR, MacKowiak SD, Li N, Chen W, Rajewsky N. miRDeep2 accurately identifies known and hundreds of novel microRNA genes in seven animal clades. Nucleic Acids Res. 2012;40:37-52.

28. Bolstad B. preprocessCore: A collection of pre-processing functions. R package version 1.50.0 [Internet]. 2020. Available from: https://github.com/bmbolstad/preprocessCore.

29. Storey J, Leek J, Bass A. EDGE: extraction of differential gene expression. R package version 2.20.0. 2020. Available from: https://github.com/StoreyLab/edge.

30. Awamleh Z, Gloor GB, Han VKM. Placental microRNAs in pregnancies with early onset intrauterine growth restriction and preeclampsia: Potential impact on gene expression and pathophysiology. BMC Med Genomics BMC Medical Genomics. 2019;12:1-10.

31. Rubio M, Bustamante M, Hernandez-Ferrer C, Fernandez-Orth D, Pantano L, Sarria Y, et al. Circulating miRNAs, isomiRs and small RNA clusters in human plasma and breast milk. PLoS One. 2018;13:119.

32. Kiserud T, Benachi A, Hecher K, Perez RG, Carvalho J, Piaggio G, et al. The World Health Organization fetal growth charts: concept, findings, interpretation, and application. Am J Obstet Gynecol. 2018;218:619-29.

33. Morales-Prieto DM, Chaiwangyen W, Ospina-Prieto S, Schneider U, Herrmann J, Gruhn B, et al. MicroRNA expression profiles of trophoblastic cells. Placenta. 2012;33:725-34.

34. Alter O, Brown PO, Botstein D. Singular value decomposition for genome-Wide expression data processing and modeling. Proc Natl Acad Sci U S A. 2000;97:10101-6. 
35. Tsui NBY, Jiang P, Wong YF, Leung TY, Allen Chan KC, Chiu RWK, et al. Maternal plasma RNA sequencing for genome-wide transcriptomic profiling and identification of pregnancy-associated transcripts. Clin Chem. 2014;60:954-62.

36. Li H, Guo L, Wu Q, Lu JF, Ge Q, Lu Z. A comprehensive survey of maternal plasma miRNAs expression profiles using high-throughput sequencing. Clin Chim Acta [Internet]. Elsevier B.V.; 2012;413:568-76. Available from: http://dx.doi.org/10.1016/j.cca.2011.11.026.

37. Pan M, Ge Q, Li H, Yang Q, Lu J, Zhang D, et al. Sequencing the MiRNAs in maternal plasma from women before and after parturition. J Nanosci Nanotechnol. 2012;12:4035-43.

38. Renthal NE, Chen C-C, Williams KC, Gerard RD, Prange-Kiel J, Mendelson CR. miR-200 family and targets, ZEB1 and ZEB2, modulate uterine quiescence and contractility during pregnancy and labor. Proc Natl Acad Sci. 2010;107:20828-33.

39. Liang $Y$, Ridzon D, Wong $L$, Chen C. Characterization of microRNA expression profiles in normal human tissues. BMC Genom. 2007;8:1-20.

40. Bentwich I, Avniel A, Karov Y, Aharonov R, Gilad S, Barad O, et al. Identification of hundreds of conserved and nonconserved human microRNAs. Nat Genet. 2005;37:766-70.

41. Lin S, Cheung WKC, Chen S, Lu G, Wang Z, Xie D, et al. Computational identification and characterization of primate-specific microRNAs in human genome. Comput Biol Chem [Internet]. Elsevier Ltd; 2010;34:232-41. Available from: http://dx.doi.org/10.1016/j.compbiolchem.2010.08.001.

42. Zhang R, Wang YQ, Su B. Molecular evolution of a primate-specific microRNA family. Mol Biol Evol. 2008;25:1493-502.

43. Chang G, Mouillet JF, Mishima T, Chu T, Sadovsky E, Coyne CB, et al. Expression and trafficking of placental microRNAs at the feto-maternal interface. FASEB J. 2017;31:2760-70.

44. Chim SSC, Shing TKF, Hung ECW, Leung TY, Lau TK, Chiu RWK, et al. Detection and characterization of placental microRNAs in maternal plasma. Clin Chem. 2008;54:482-90.

45. Salem NA, Mahnke AH, Wells AB, Tseng AM, Yevtushok L, Zymak-Zakutnya N, et al. Association between fetal sex and maternal plasma microRNA responses to prenatal alcohol exposure: Evidence from a birth outcome-stratified cohort. Biol Sex Differ Biology of Sex Differences. 2020;11:1-17.

46. Chakrabarty A, Tranguch S, Daikoku T, Jensen K, Furneaux H, Dey SK. MicroRNA regulation of cyclooxygenase-2 during embryo implantation. Proc Natl Acad Sci U S A. 2007;104:15144-9.

47. Kamity R, Sharma S, Hanna N. MicroRNA-mediated control of inflammation and tolerance in pregnancy. Front Immunol. 2019;10.

48. Williams KC, Renthal NE, Gerard RD, Mendelson CR. The microRNA (miR)-199a/214 cluster mediates opposing effects of progesterone and estrogen on uterine contractility during pregnancy and labor. Mol Endocrinol. 2012;26:1857-67.

49. Dennis Lo YM, Corbetta N, Chamberlain PF, Rai V, Sargent IL, Redman CWG, et al. Presence of fetal DNA in maternal plasma and serum. Lancet. 1997;350:485-7. 
50. Khorram Khorshid HR, Zargari M, Sadeghi MR, Edallatkhah H, Shahhosseiny MH, Kamali K. Early fetal gender determination using real-time PCR analysis of cell-free fetal DNA during 6th-10th weeks of gestation. Acta Med Iran. 2013;51:209-14.

51. Strutz J, Cvitic S, Hackl H, Kashofer K, Appel HM, Thüringer A, et al. Gestational diabetes alters microRNA signatures in human feto-placental endothelial cells depending on fetal sex. Clin Sci. 2018;132:2437-49.

\section{ADDITIONAL FILE LEGENDS.}

53. Additional. file 1. Reproductive samples-associated c-miRNA signatures. c-miRNA signatures were defined here as the top $25 \%$ most highly expressed in each available miRNA-seq expression data in placenta, umbilical cord plasma, amniotic fluid, and early breast milk (48h after birth) obtained from the NCBI Gene Expression Omnibus (accession numbers: GSE114349, GSE112343, GSE107524; $(6,24,25)$. All these data were analyzed by paired Wilcoxon tests in plasma of pregnant women, relative to their expression in non-pregnant control women. P: placenta, AF: amniotic fluid, UC: umbilical cord plasma, BM: breast milk.

54. Additional. file 2. Similarities and differences between reproductive samples-associated c-miRNA signatures. Venn diagram illustrating c-miRNA signatures overlapping (similarities) and nonoverlapping (difference) between normal placenta, amniotic fluid, umbilical cord plasma, and breast milk.

\section{Figures}




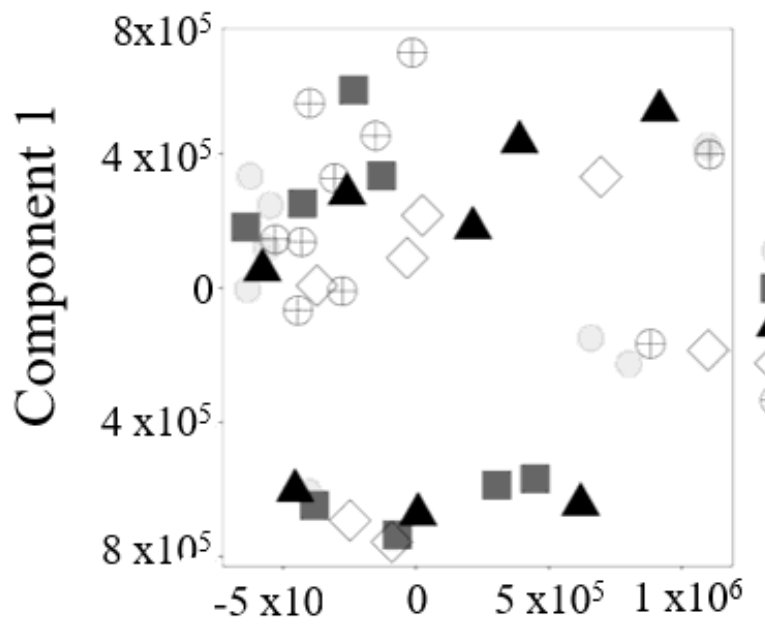

Component 2

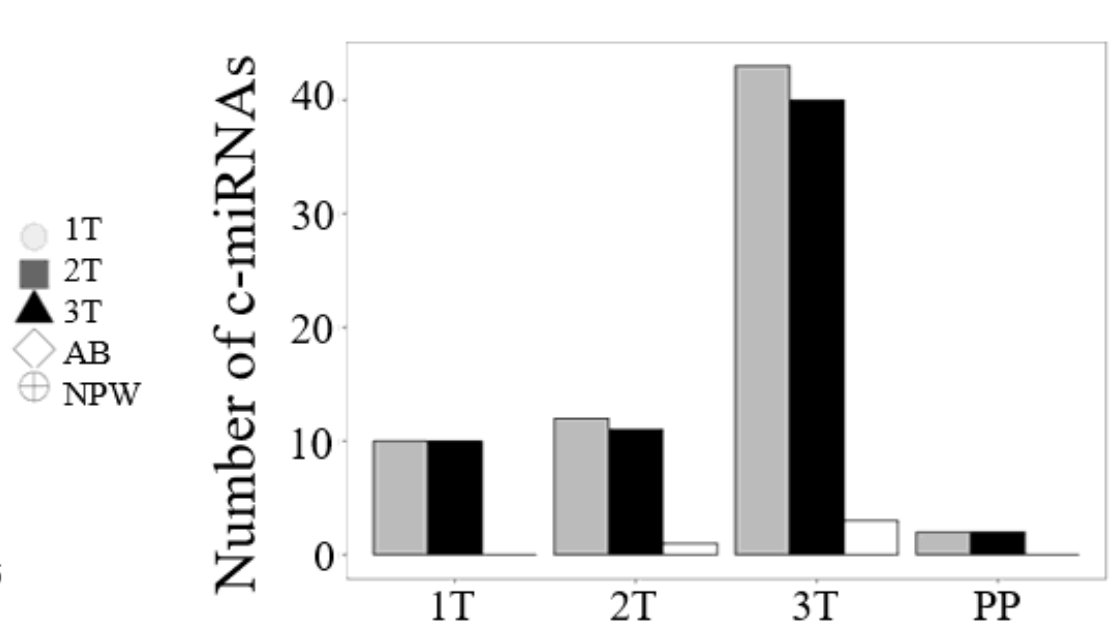

Total $\square$ Overexpression $\square$ Subexpression

D
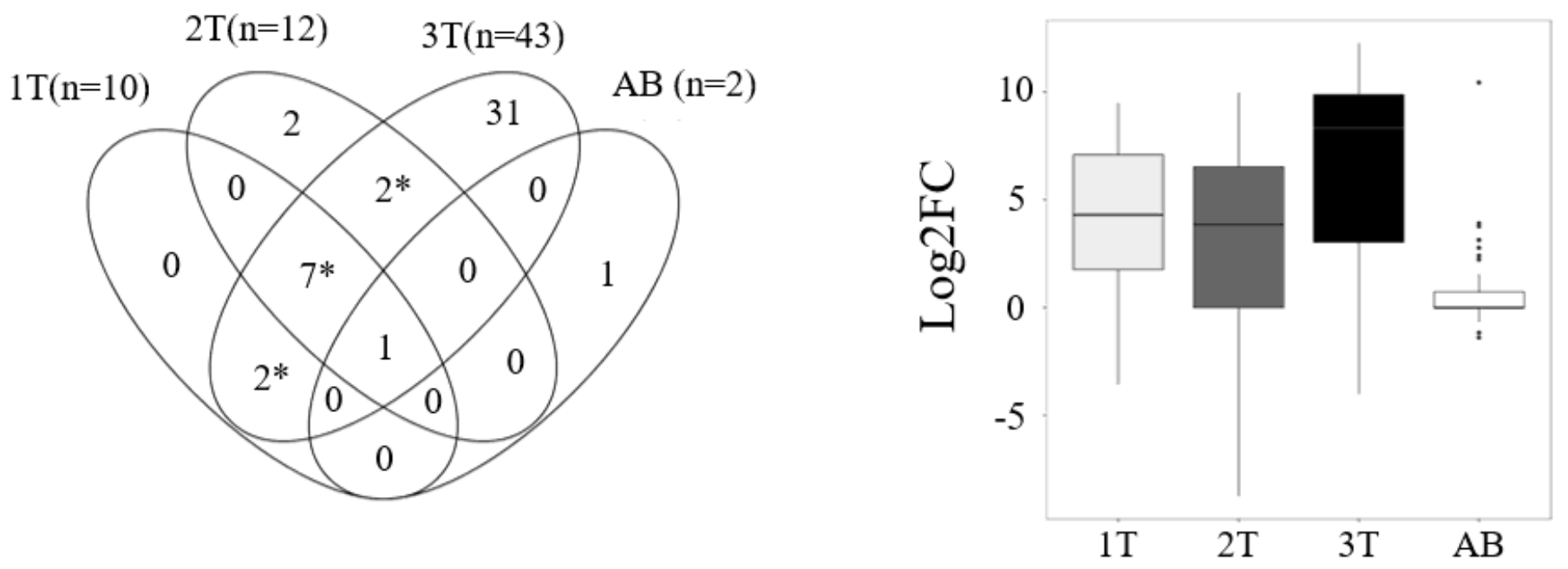

Figure 1

Global comparison of c-miRNA expression profile throughout pregnancy. A) Global similarity in normalized c-miRNA expression profiles between both individual pregnant and non-pregnant women, as well as across stages, being revealed by multidimensional scaling analysis. B) Bar chart indicating the total number of differentially expressed c-miRNAs (grey), overexpressed (black) and sub expressed (white) when comparing pregnant women at each indicated period with non-pregnant control subjects. C) Venn diagram illustrating overlaps of differentially expressed c-miRNAs across all four stages. Asterisks indicate significance in overlap (Fisher exact test $p<0.005$ ). D) Boxplot charts show increasing 
expression level among differentially expressed c-miRNAs during $1 \mathrm{~T}, 2 \mathrm{~T}, 3 \mathrm{~T}$, and a subsequent drop at $\mathrm{AB}$. (1T: first trimester of pregnancy, 2T: second, 3T: third, AB: after birth).

$\mathbf{A}$

\section{C14CM}

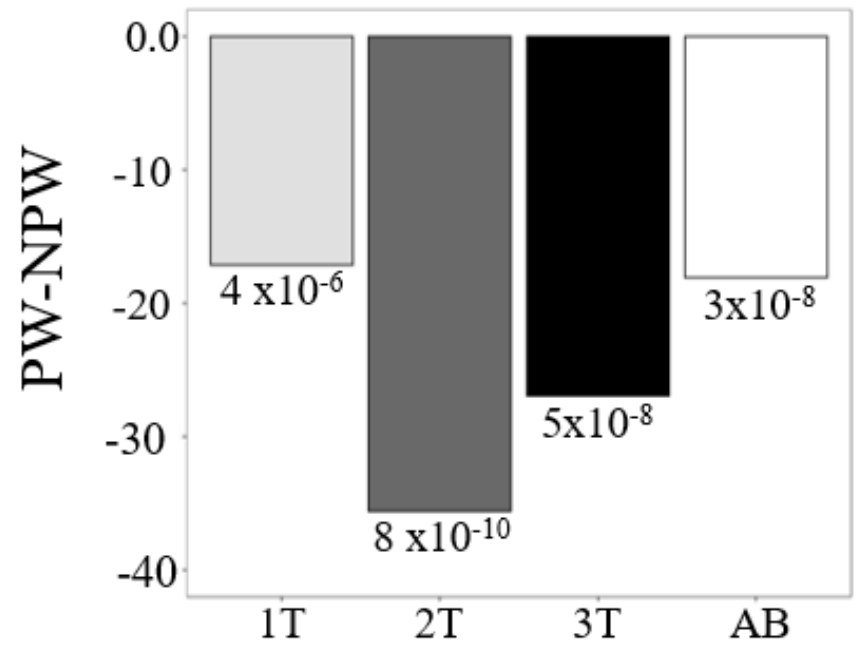

B

C19CM

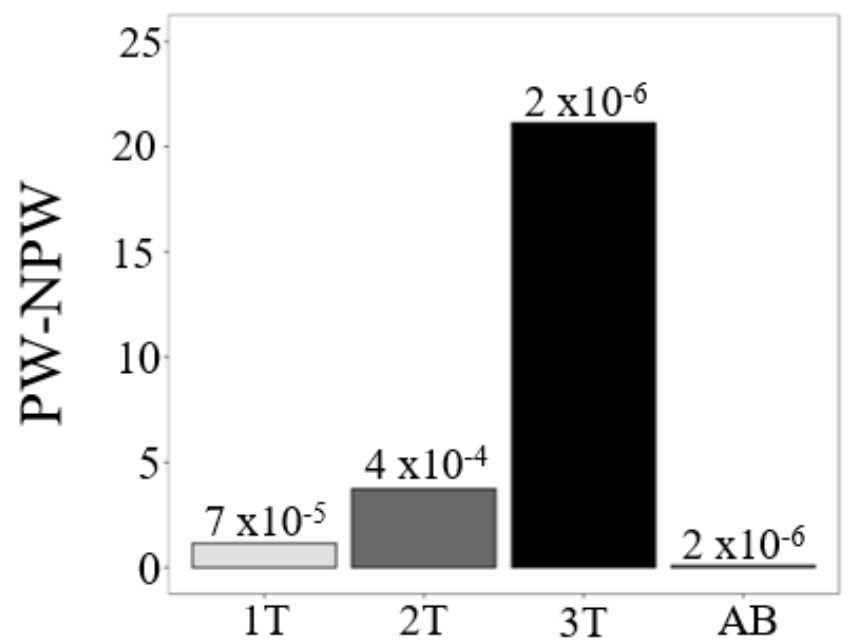

\section{Figure 2}

Trophoblast-associated miRNA families expressed in the plasma of pregnant women. A) Bar chart showing median difference in the expression of C14MC $(n=84)$ between pregnant and non-pregnant samples at each indicated stage during and after pregnancy. B) Bar chart showing median difference in expression of the $\mathrm{C19MC}(\mathrm{n}=54)$ between pregnant and non-pregnant samples at each indicated stage during and after pregnancy. $P$ values for each comparison (paired Wilcoxon paired test) are indicated for each individual comparison. 
A

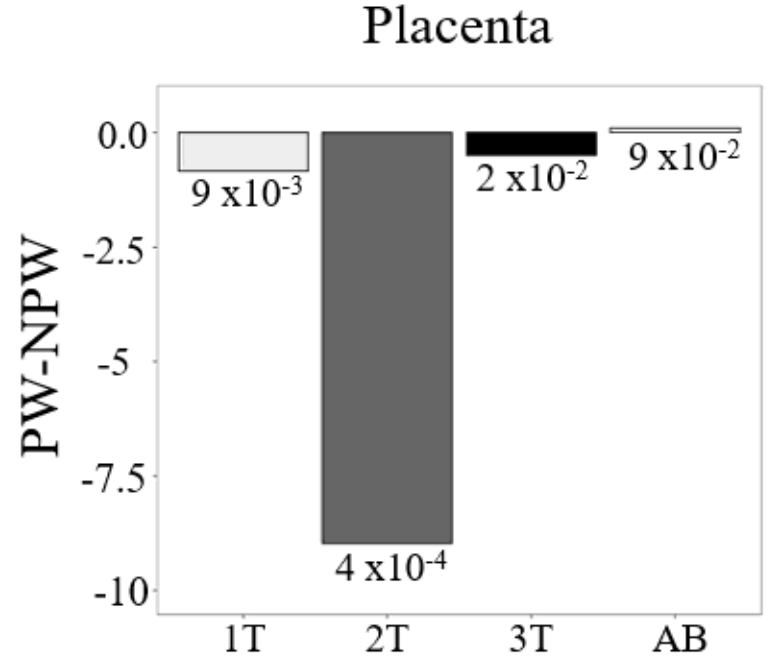

C

Umbilical cord plasma

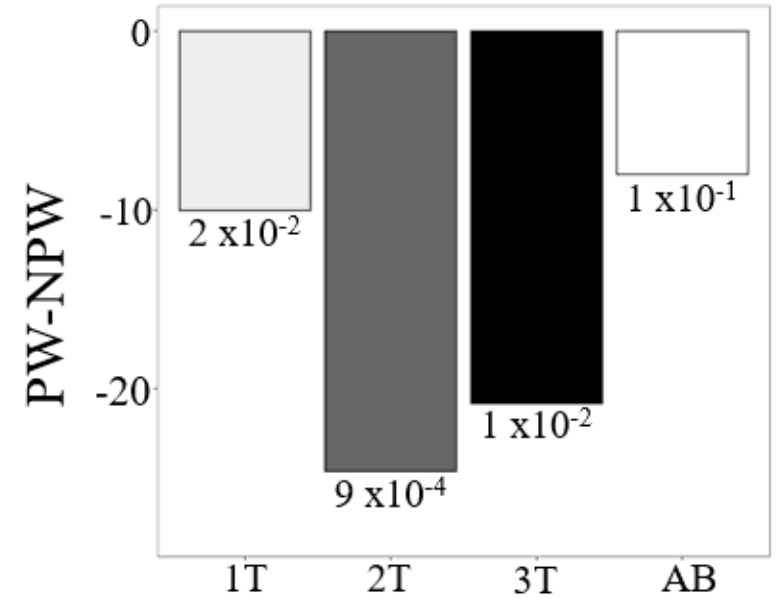

B

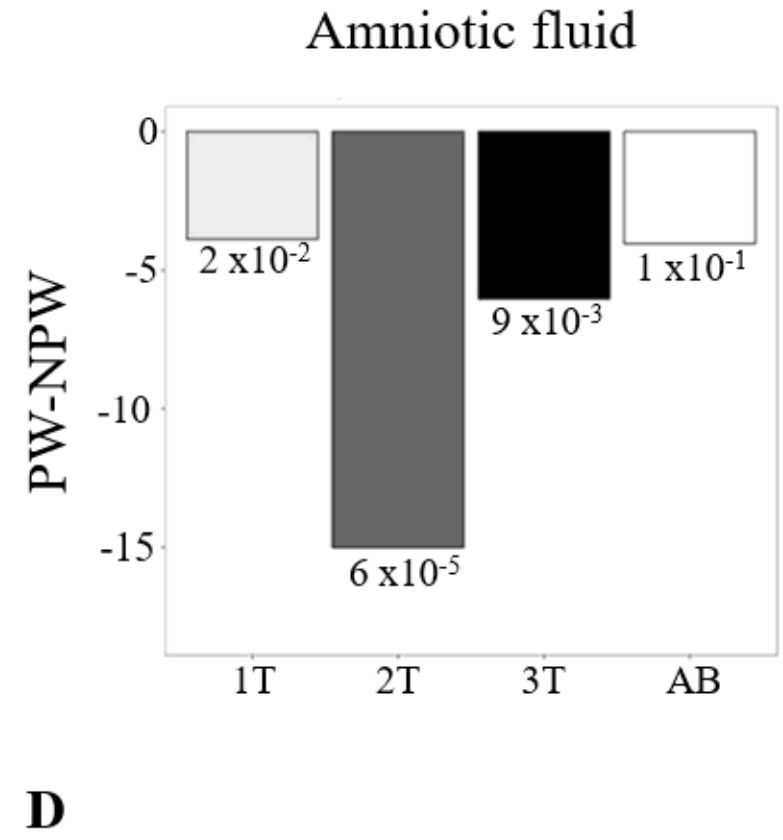

Breast milk

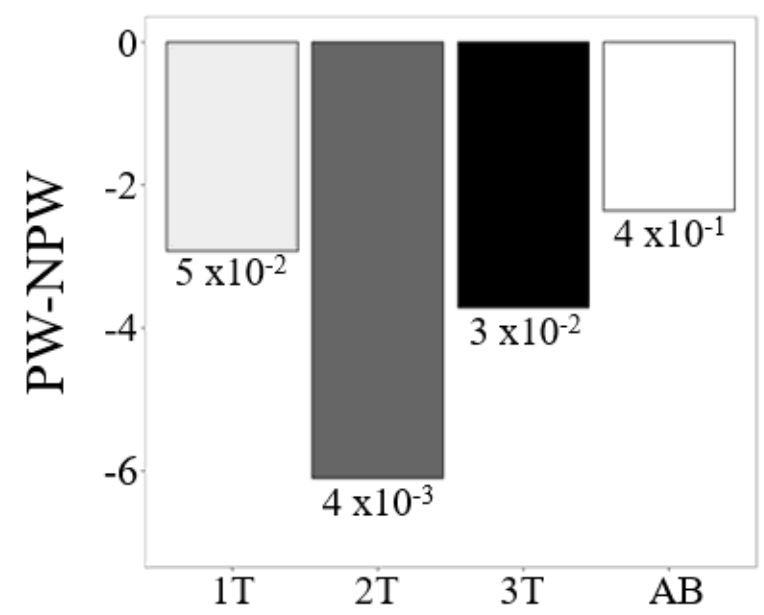

\section{Figure 3}

Down regulation of c-miRNAs most highly expressed in placenta, amniotic fluid, umbilical cord plasma, and breast milk in plasma of pregnant women at four different stages during and after pregnancy. The expression of the top $25 \%$ most highly expressed miRNAs in each of the indicated tissues and fluids was measured in maternal peripheral plasma and compared against that of non pregnant women by paired Wilcoxon test. A) Bar chart showing the median difference in expression of c-miRNAs most highly expressed in placenta $(n=362)$ at each indicated stage. B) Bar chart showing the median difference in expression of c-miRNAs most highly expressed in amniotic fluid $(n=362)$. C) Bar chart showing median difference in expression of c-miRNAs most highly expressed in umbilical cord plasma $(n=362)$. D) Bar 
chart sowing mean difference in expression of c-miRNAs most highly expressed in breast milk $(n=362)$ at each indicated stage. 1T, 2T, 3T and AB: first trimester, second trimester, third trimester and after birth, respectively. $\mathrm{P}$ values (paired Wilcoxon signed rank test) are indicated under each bar.

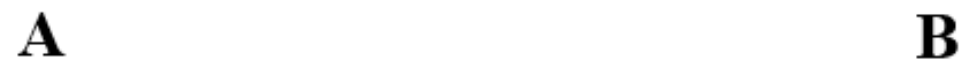

First trimester

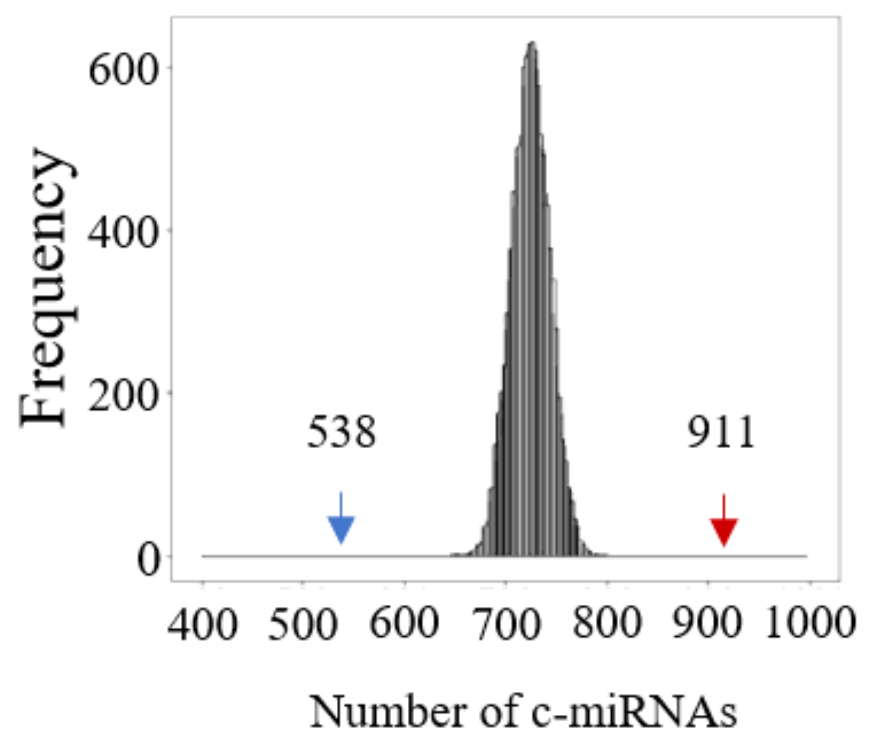

C

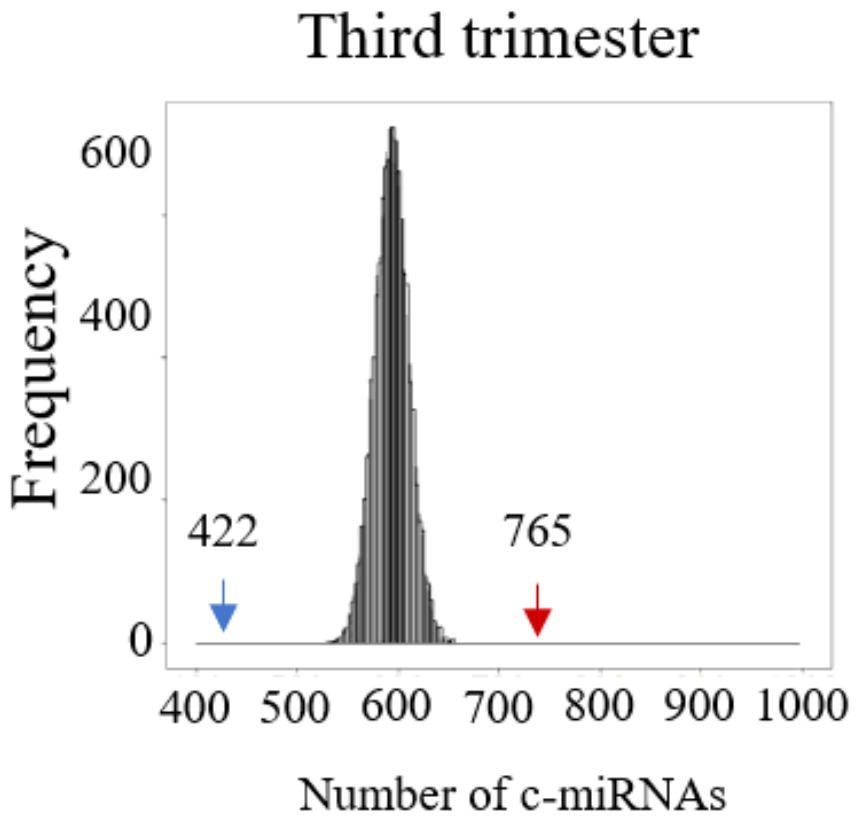

B
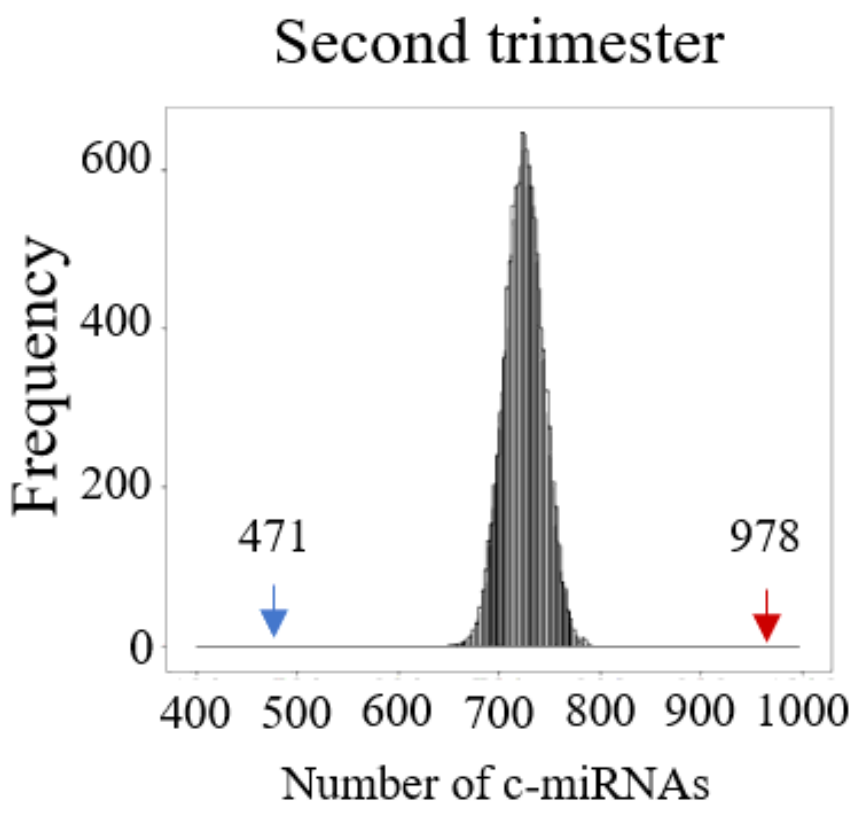

D
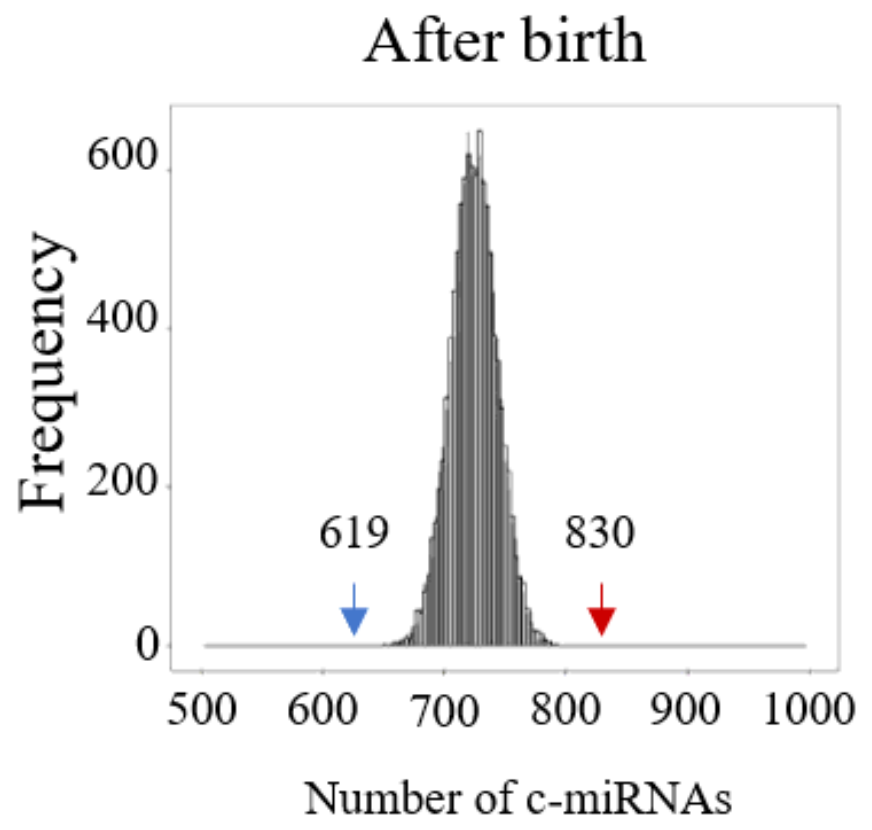

Figure 4

Biased expression in maternal c-miRNAs associated to fetal sex during pregnancy. Differences in expression in c-miRNAs between women giving birth to female babies relative to women giving birth to 
male babies were calculated and the total number of transcripts increasing their expression was obtained for each pregnancy period regardless of statistical significance at the level of individual miRNAs. A-D) Observed number of up (red arrow) and down (blue arrow) regulated miRNAs for each indicated trimester compared with the expected distribution of both up or down regulated miRNAs estimated using 10,000 randomizations of expression values per gene across samples for each separate time period. Arrows located outside the expected distribution indicate a $\mathrm{p}$ value $<0.0001$.

\section{Fetal weight}

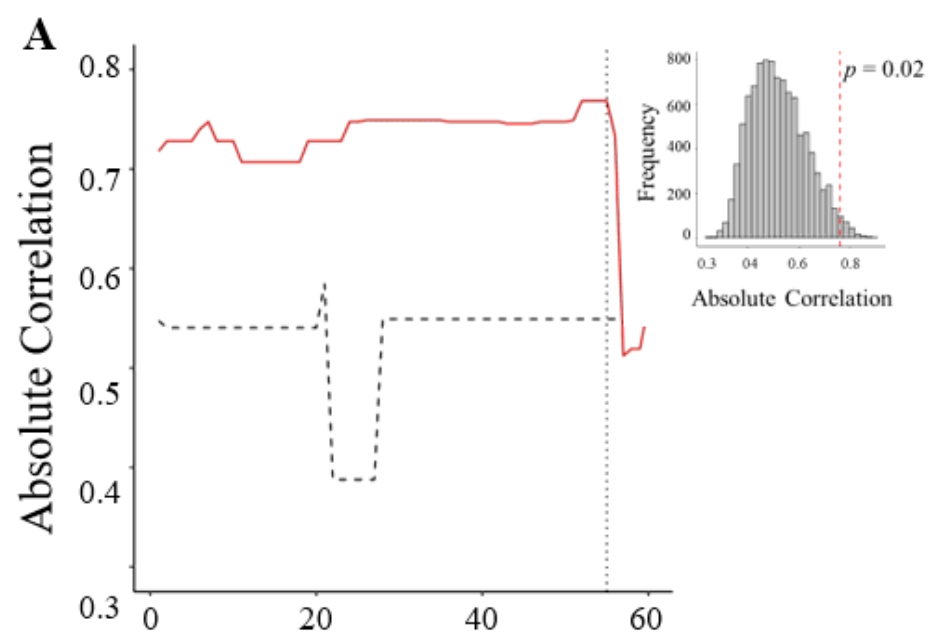

Number of top c-miRNAs

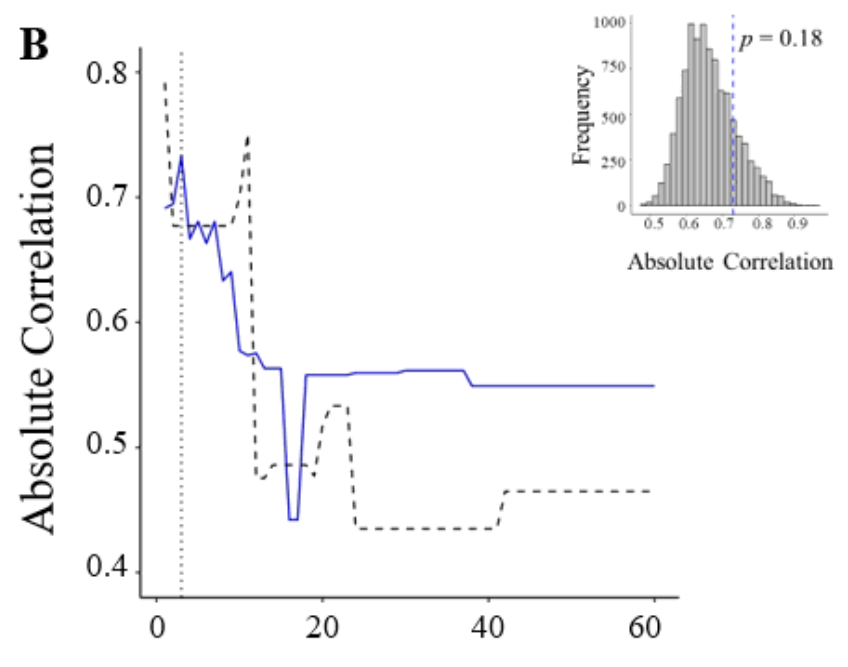

Number of top c-miRNAs

\section{Femur length}
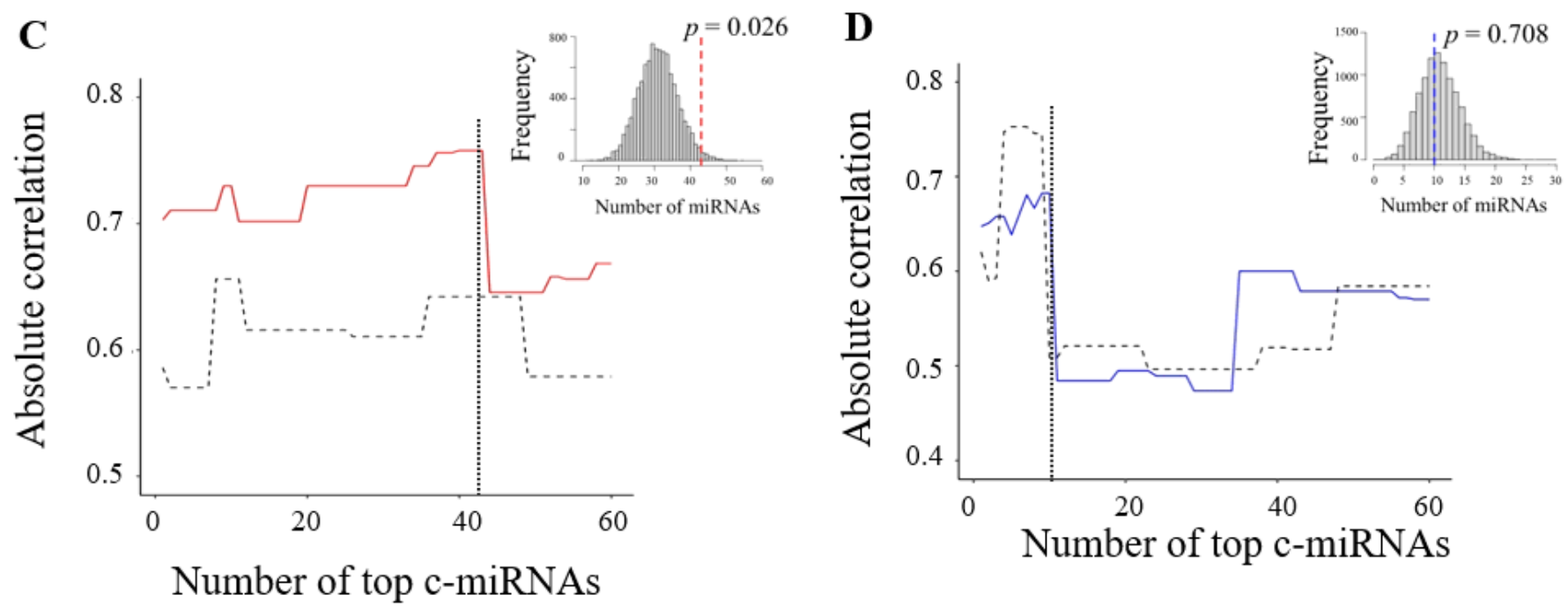

Figure 5 
c-miRNA signatures of fetal growth throughout pregnancy. Spearman correlations between fetal growth indicators and transcript abundance were calculated for every miRNA present in maternal circulation across pregnancy samples. A sequence of partial signatures was created by successively adding each cmiRNA in descending order of correlation with fetal weight and fetal length, starting from the topmost highly correlated transcript. Expression of each successive signature was summarized by their associated eigengene and correlated with both fetal growth indicators to quantify its strength of association. Left column charts show absolute Spearman correlation with fetal weight (A) and femur length (C) for each successive signature as additional c-miRNAs added in descending order of correlation starting from the topmost highly correlated transcript (red line). Right column charts show the strength of association with fetal weight (B) and femur length (D) for each successive signature as additional miRNAs added in ascending order of absolute correlation starting from the most negatively correlated transcript (blue line). Dashed lines in charts represent the same analysis carried out after expression was randomized for each transcript across all samples. The optimal signature is indicated by the vertical line. Insets show the probability of obtaining a similarly correlated optimal signature after 10,000 independent randomizations of the original expression data. 


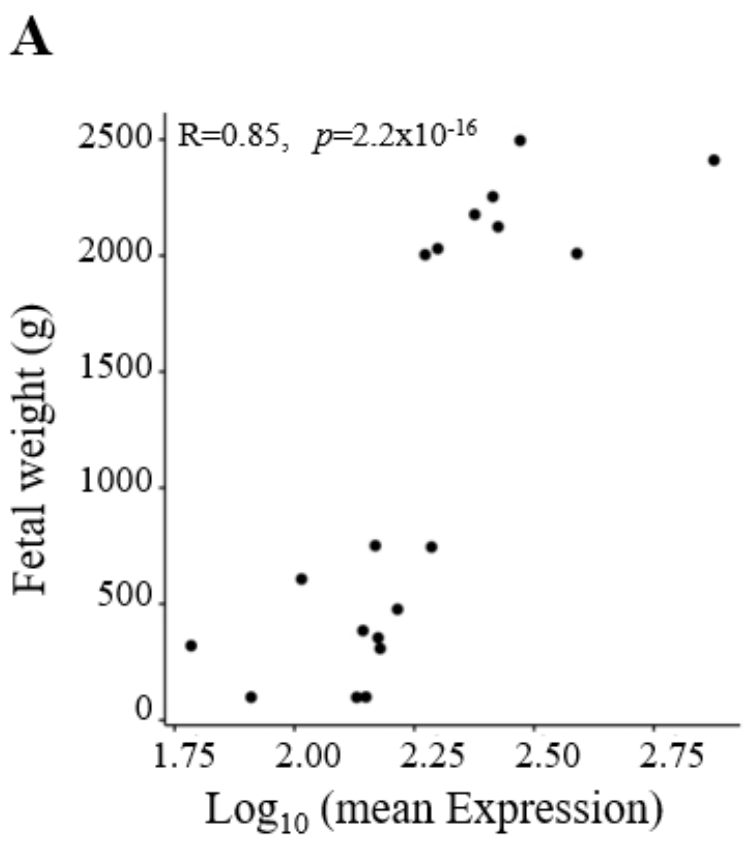

C

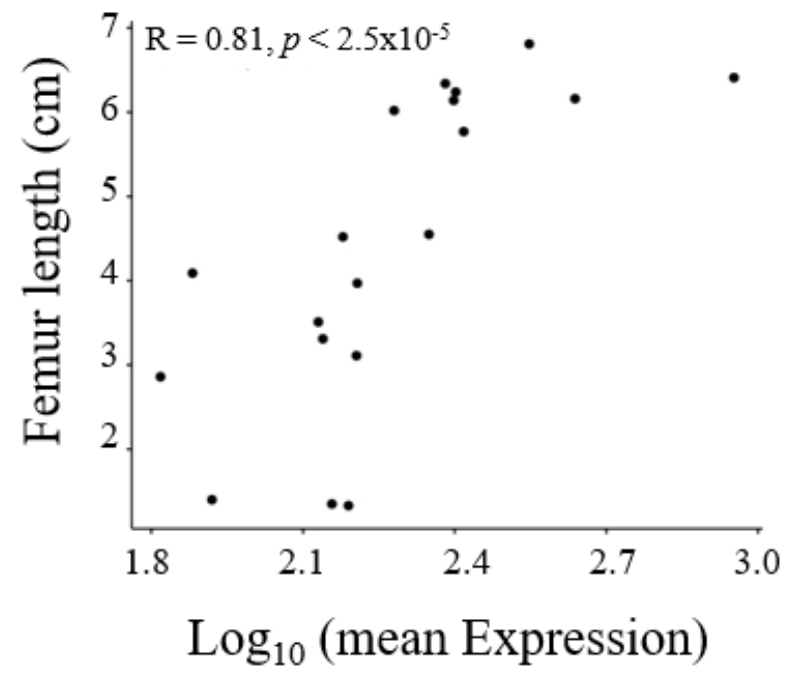

B

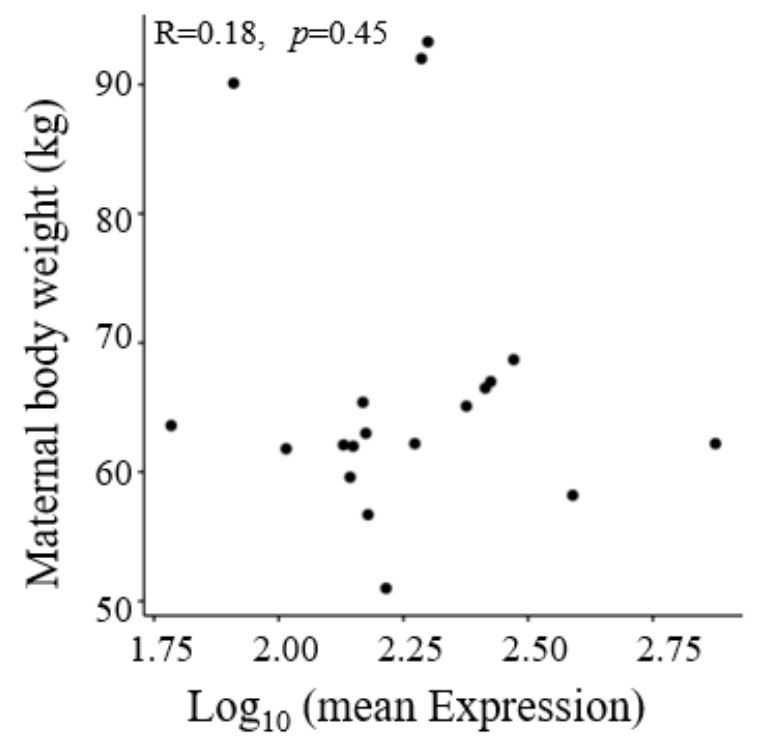

D

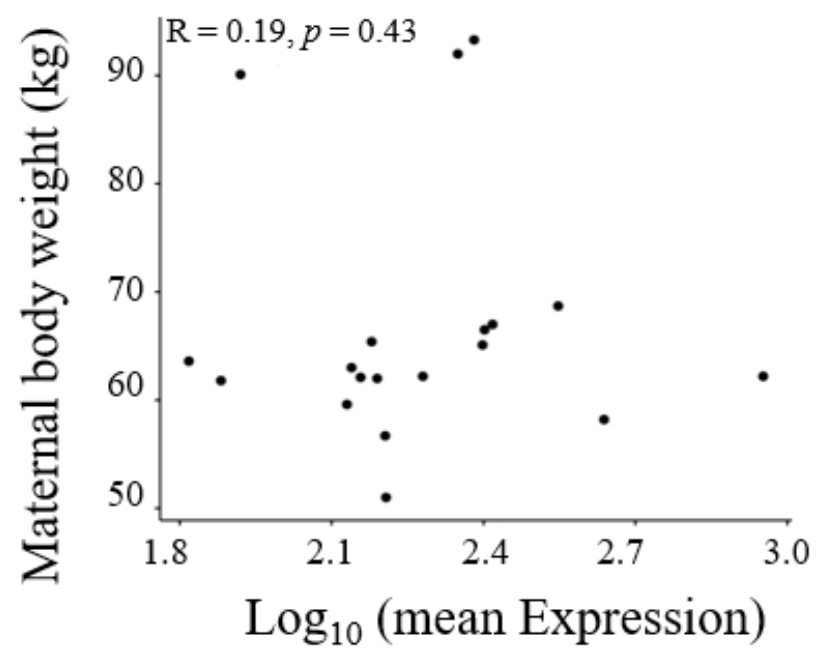

\section{Figure 6}

Maternal c-miRNA fetal growth signatures are not associated to maternal changes in body weight. Optimal signatures of fetal growth (fetal weight $(n=56)$ and femur length $(n=43))$ were summarized by its mean expression across samples and correlated with either both indicators of fetal growth or maternal body weight data obtained from the exact same women. Scatter graphs in the left column display the association between fetal weight (A) or femur length (C) and the mean expression of the identified optimal signature. Scatter graphs in the right column display the association between maternal body 
weight and the mean expression of the optimal signature identified in Figure 5A and C. Spearman Correlation rho and $\mathrm{p}$ values for each comparison are indicated.

\section{Supplementary Files}

This is a list of supplementary files associated with this preprint. Click to download.

- Additionalfile2.tif

- Additionalfile1.tif 